\begin{tabular}{|c|}
\hline Research \\
\hline \\
\hline
\end{tabular}

\title{
Reverse Unfolding of Petri Nets and its Application in Program Data Race Detection
}

\author{
Zongyin Hao (郝宗寅 $)^{1,2}$, Faming Lu (鲁法明 $)^{1}$ \\ 1 (College of Computer Science and Engineering, Shandong University of Science and Technology, Qingdao \\ 266590, China) \\ 2 (School of Informatics, Xiamen University, Xiamen 361005, China) \\ Corresponding author: Faming Lu, fm_lu@163.com
}

\begin{abstract}
The unfolding technique can partially alleviate the state explosion in Petri nets through branching processes. However, all states of a system are still contained in its unfolding net. To deal with some practical problems, only the coverability determination of a specific state is needed. In view of this, reducing the scale of the unfolding net is feasible. This study proposes a target-oriented reverse unfolding algorithm for the coverability determination of 1-safe Petri nets, which combines a heuristic technique to reduce the scale of unfolding nets, thereby improving the efficiency of coverability determination. Furthermore, the reverse unfolding is applied to the formal verification of concurrent programs, and their data race detection is converted into the coverability determination of a specific state in 1-safe Petri nets. The experiment compares the efficiency between forward unfolding and reverse unfolding in the coverability determination of a Petri net. The results show that when the Petri net has more forward branches than backward branches, reverse unfolding is more efficient than forward unfolding. Finally, the key factors influencing the efficiency of reverse unfolding are analyzed.
\end{abstract}

Keywords Petri nets; coverability determination; reverse unfolding; heuristic optimization; data race detection

Citation Hao ZY, Lu FM. Reverse unfolding of Petri nets and its application in program data race detection, International Journal of Software and Informatics, 2021, 11(4): 405-428. http://www.ijsi.org/ 1673-7288/254.htm

As a modeling and analysis tool for distributed concurrent systems, Petri nets have been widely used in flexible manufacturing systems ${ }^{[1,2]}$, business process management systems ${ }^{[3,4]}$, and formal verification of concurrent programs ${ }^{[5,6]}$. However, the state explosion prevents the application of Petri nets in the analysis of large-scale concurrent systems. In view of this, McMillan ${ }^{[7]}$ firstly proposed to describe system behaviors by the unfolding of nets and

This is the English version of the Chinese article “Petri 网的反向展开及其在程序数据竞争检测的应用. 软件 学报, 2021, 32(6): 1612-1630. doi: 10.13328/j.cnki.jos.006240”.

Funding items: National Natural Science Foundation of China $(61602279,61472229)$; National Key Research and Development Plan (2016YFC0801406); Taishan Scholars Program of Shandong Province (ts20190936); Excellent Youth Innovation Team Foundation of Shandong Higher School (2019KJN024); Postdoctoral Innovation Foundation of Shandong Province (201603056); Shandong-Chongqing Science and Technology Cooperation Plan (cstc2020jscxlyjsAX0008); Open Foundation of First Institute of Oceanography, MNR (2018002); Shandong University of Science and Technology Research Fund (2015TDJH102)

Received 2020-08-31; Revised 2020-10-26; Accepted 2020-12-19; IJSI published online 2021-12-23 
constructed finite complete prefixes of unfolding nets by branching processes ${ }^{[8]}$ and the partial ordering, effectively alleviating the state explosion in the property analysis of Petri nets. After that, the unfolding technique received much attention.

Esparza et al. ${ }^{[9]}$ pointed out that the partial order relation defined by McMillan led to an exponential increase in the size of finite complete prefixes in some cases. They proposed a total order relation for 1-safe Petri nets to minimize the size of finite complete prefixes. Khomenko et al. ${ }^{[10]}$ standardized the definition of unfolding and conducted the parameterization of unfolding. Heljanko et al.$^{[11]}$ parallelized the unfolding technique to improve the unfolding efficiency. Benito et al. ${ }^{[12]}$ extended the unfolding technique to timed Petri nets, and Schwarick et al..$^{[13]}$ extended it to colored Petri nets. In the application of unfolding of Petri nets, Lu et al. ${ }^{[5]}$ proposed a finite unfolding technique of unbounded Petri nets for deadlock detection of net systems. Xiang et al. ${ }^{[6]}$ detected data inconsistency in concurrent systems with the unfolding technique. Dong et al. ${ }^{[14]}$ verified Computation Tree Logic (CTL) utilizing reachability graphs of Petri nets. Liu et al. ${ }^{[15]}$ detected the robustness of workflows by branching processes. In terms of the property analysis of Petri nets, Chatain $e t$ al. ${ }^{[16]}$ designed a goal-driven unfolding technique for the coverability problem of Petri nets to prune redundant transitions by analyzing internal causalities. Bonet et al. ${ }^{[17]}$ proposed a semi-adequate ordering approach based on heuristics to improve the efficiency of the unfolding technique in the coverability analysis of Petri nets. Later, they proved that the extension order could be independent of the partial order of cut-off events ${ }^{[18]}$, which broadened the application of the heuristic unfolding technique in property analysis of Petri nets.

The unfolding of nets alleviates the state explosion in the property analysis of Petri nets to some extent by branching processes and the partial ordering. However, all the states of a system are still contained in its unfolding net. Some practical problems only require to determine the coverability of a specific state. In light of this, simplifying the net unfolding is feasible. To this end, we propose a target-oriented reverse unfolding algorithm for the coverability determination of 1-safe Petri nets. Starting from the target marking that needs coverability determination, reverse unfolding only describes the system states related to coverability determination and reduces the unfolding scale with the heuristic technique, so as to improve the determination efficiency. Further, we apply the reverse unfolding algorithm to formal verification of concurrent programs and convert their data race detection to coverability determination of specific markings in Petri nets. The experiment compares the efficiencies of heuristic reverse unfolding and directed unfolding ${ }^{[17]}$ (a forward unfolding also using the heuristic technique) in coverability determination of Petri nets. The results show that in 415 groups of test data, the scale of reverse unfolding is better than that of directed unfolding on 85 groups of data and is comparable to that of directed unfolding on 26 groups of data. At last, we analyze and summarize the key factors influencing the efficiency of reverse unfolding.

Focusing on the coverability determination of Petri nets, we analyze the application scenarios of forward unfolding and reverse unfolding and use examples to illustrate the advantages of reverse unfolding over forward unfolding in the first section. In the second section, we introduce the reverse unfolding algorithm of Petri nets, including the basic definitions, the algorithm flow, and the heuristic optimization strategy. In Section 3, we apply reverse unfolding to data race detection of concurrent programs. In Section 4, we use experiments to evaluate the efficiency of forward unfolding and reverse unfolding in coverability determination of Petri nets. In Section 5 , we make a summarization and a prospect. 


\section{Examples and Motivation Analysis}

In this section, we first discuss the application scenarios of forward unfolding and reverse unfolding in coverability determination of Petri nets. Then we illustrate the advantages of reverse unfolding over forward unfolding by an example.

Here are two simple examples. In Figure 1(a), the initial marking of the Petri net is $\{p s\}$, and the coverability of the target marking $\{p t\}$ should be verified. For simplicity of presentation, the path on the left side in Figure 1(a) is called path1, and that on the right side is called path2. In this example, the forward unfolding starts from the initial marking $\{p s\}$, and it has difficulty in selecting path 1 or path2. If it unfortunately selects path 2 , it will make plenty of redundant extensions. Unlike the forward unfolding, the reverse unfolding starts from the target marking $\{p t\}$ and only needs to extend reversely along path 1 . Thus, it can easily find a reachable path to the initial marking $\{p s\}$.

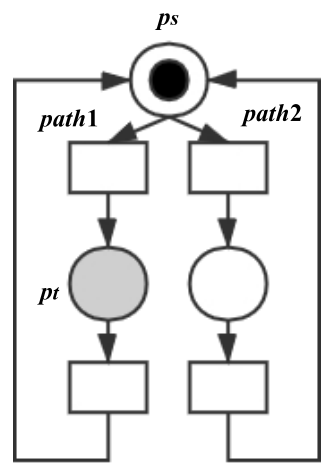

(a) Application scenario of reverse unfolding

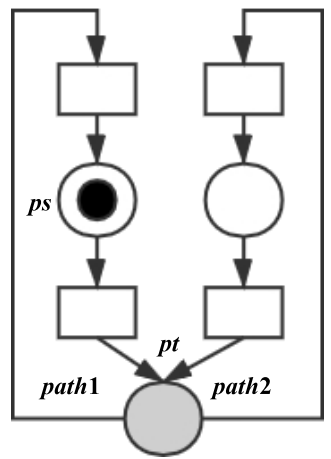

(b) Application scenario of forward unfolding

Figure 1 Application scenarios of forward unfolding and reverse unfolding

Figure 1(b) can be seen as an "inversion" of Figure 1(a). In this example, the reverse unfolding starts from the target marking $\{p t\}$, and it has difficulty in selecting path 1 or path2; while the forward unfolding starts from the initial marking $\{p s\}$, and it easily reaches the target marking $\{p t\}$ along path1.

As indicated by the above two examples, reverse unfolding is suitable to Petri nets that have more forward branches. When a Petri net has more reverse branches, the forward unfolding is more applicable. Specifically, forward unfolding starts from the initial marking of a Petri net to describe the system, which implies the system's complete behaviors; while reverse unfolding starts from the target marking whose coverability needs to be determined and only describes the system states related to coverability determination. In this paper, we design and realize the reverse unfolding algorithm from this perspective.

Then, we use an example to further illustrate the advantages of reverse unfolding over forward unfolding. In Figure 2(a), the initial marking of the Petri net is $\{p 1\}$, and the coverability of the target marking $\{p 10\}$ needs to be verified. Figure 2(b) shows the forward unfolding of the Petri net ${ }^{[9]}$, and Figure 2(c) shows its reverse unfolding. The two both use the adequate order, which is based on the breadth-first strategy, as the extension order. Once the coverability of the target marking is verified, the extension ends. In this example, the forward unfolding generates 19 nodes and 20 flow relations. The reverse unfolding yields 14 nodes and 14 flow relations. The scale of the reverse unfolding is better than that of the forward unfolding. This is because the 
contribution of the transition $\{t 1, t 3, t 4, t 7\}$ to the coverability of $\{p 10\}$ is redundant (namely that the path on the left is redundant). The forward unfolding implies complete behaviors of the system, and it inevitably analyzes the redundant behaviors of $\{t 1, t 3, t 4, t 7\}$. Regarding the reverse unfolding, only the system states related to coverability determination are described, which avoids the redundant description of $\{t 3, t 7\}$. Although the reverse unfolding still includes redundancies, its overall scale is better than that of forward unfolding.

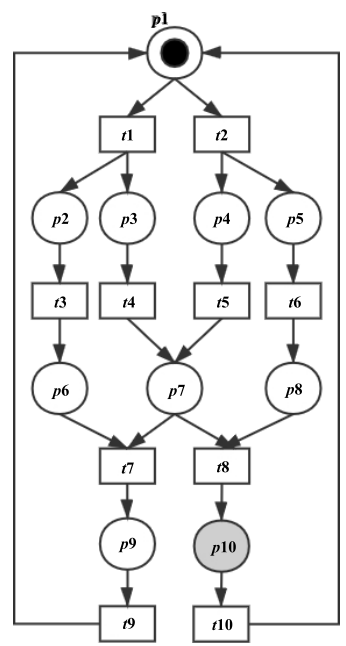

(a) Petri net

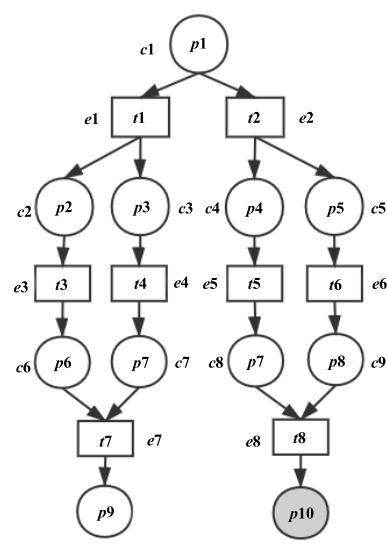

(b) Forward unfolding of Petri net

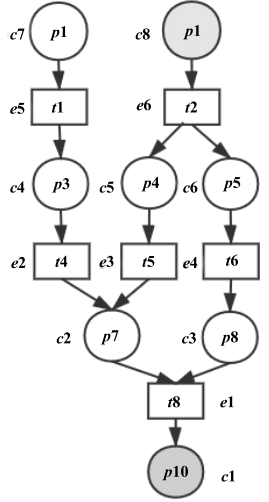

(c) Reverse unfolding of Petri net

Figure 2 An example manifesting the advantage of reverse unfolding over forward unfolding

In this section, we discuss the advantages of forward unfolding and reverse unfolding. It should be noted that these advantages are generalized as they are determined by properties of algorithms. For forward unfolding such as goal-driven unfolding ${ }^{[16]}$ and directed unfolding ${ }^{[17]}$, although they can use internal causalities to prune or use heuristics to improve the algorithm efficiency, they inevitably analyze redundant system behaviors when there are more forward branches. Similarly, even if reverse unfolding is equipped with the heuristic technique, it cannot assure the algorithm efficiency when there are more reverse branches. Under this premise, we further illustrate that reverse unfolding outperforms the forward unfolding in some cases through examples. Then we will introduce the reverse unfolding algorithm in detail.

\section{Reverse Unfolding of Petri Nets}

\subsection{Concept of reverse unfolding}

\subsubsection{Petri nets}

A net can be defined as a triple $(P, T, F)$, where $P$ is a place set, $T$ a transition set, $F$ the flow relation between $P$ and $T$ with $F \subset(P \times T) \cup(T \times P)$. We define the preset and post set of the node $x$ as ${ }^{\bullet} x=\{y \in P \cup T \mid F(y, x)=1\}$ and $x^{\bullet}=\{y \in P \cup T \mid F(x, y)=1\}$, respectively. The marking of the net $(P, T, F)$ is a multiset established on $P$. In graphical representation, we present the markings of a net by adding the corresponding number of tokens to each place.

A net system can be defined as a quadruple $\left(P, T, F, M_{0}\right)$, where $M_{0}$ is the initial marking of the net $(P, T, F)$. If $\forall p \in P: F(p, t) \leq M(p)$, the transition $t$ under the marking $M$ 
enables, and the enabled transition is executable. The execution of $t$ makes the system enter into a new marking $M^{\prime}$, which is denoted as $M \stackrel{t}{\rightarrow} M^{\prime}$, i.e., for each place $p, M^{\prime}(p)=$ $M(p)-F(p, t)+F(t, p)$. A transition sequence $\sigma=t_{1} t_{2} \cdots t_{n}$ is denoted as a triggered sequence when and only when there are markings $M_{1}, M_{2}, \cdots, M_{n-1}, M_{n}$ satisfying $M_{0} \stackrel{t_{1}}{\longrightarrow}$ $M_{1} \stackrel{t_{2}}{\longrightarrow} \cdots \stackrel{t_{n-1}}{\longrightarrow} M_{n-1} \stackrel{t_{n}}{\longrightarrow} M_{n}$. The above formula can also be indicated as $M_{0} \stackrel{\sigma}{\rightarrow} M_{n}$, and $M_{n}$ is a reachable marking for the triggered sequence $\sigma$.

For the marking $M_{f}$, if there are markings $M$ and $M^{\prime}$ as well as the triggered sequence $\sigma$ satisfying $M \stackrel{\sigma}{\rightarrow} M^{\prime} \wedge M_{f} \subseteq M^{\prime}$, it is called that $M_{f}$ can be covered by $M$, which is denoted as $M \mapsto M_{f}$.

If the reachable marking $M$ satisfies $\forall p: M(p) \leq n, M$ is called $n$-safe. A net system is $n$-safe when and only when all of its reachable markings are $n$-safe. Particularly, the 1 -safe net systems are called as safe net systems. In this paper, we only focus on the coverability problem of 1-safe Petri nets and denote the target marking of coverability determination as $M_{f}$, namely that we verify whether $M_{0} \mapsto M_{f}$ holds.

\subsubsection{Reverse occurrence nets}

Definition 1 (Reverse occurrence nets). Reverse occurrence nets are a subclass of occurrence nets, which are used to determine the coverability of the target marking $M_{f}$ in a Petri net. A reverse occurrence net is corresponding to a quadruple $R O N=\left(C, E, F^{\prime}, C M_{f}\right)$, where $C$ is a condition set, with each condition corresponding to a token of place in the Petri net; $E$ is an event set, with each event corresponding to an execution of a transition in the Petri net; $F^{\prime}$ is the flow relation between $C$ and $E$, which corresponds to the flow relation of the Petri net; and $C M_{f}$ is the corresponding condition set of the target marking $M_{f}$ of the Petri net in $R O N$ and satisfies $\forall c \in C M_{f}: c^{\bullet}=\varnothing$.

With the Petri net shown in Figure 3(a) and the target marking $M_{f}=\{p 4\}$ as an example, its corresponding reverse occurrence net is present in Figure 3(b), where $C M_{f}=\{c 1\}$.

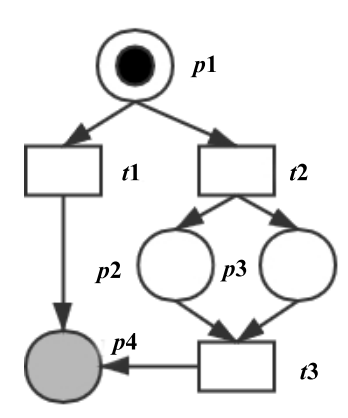

(a) Petri net

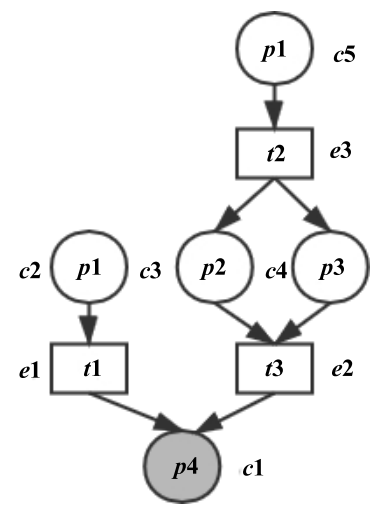

(b) Reverse occurrence net

Figure 3 An example of Petri net and its reverse occurrence net

There are three relationships between two nodes $x_{1}$ and $x_{2}$ in RON.

(1) Reverse causality: If a path starting from $x_{1}$ can reach $x_{2}$, it is denoted as $x_{2} \leq x_{1}$. Particularly, for a node $x$, there is $x=x$. In Figure 3(b), there are $c 1 \leq c 5$ and $e 2 \leq e 3$.

(2) Reverse conflict: If there are two different events $e_{1}, e_{2} \in E, e_{1}^{\bullet} \cap e_{2}^{\bullet} \neq \varnothing \wedge e_{1} \leq$ $x_{1} \wedge e_{2} \leq x_{2}, x_{1}$ and $x_{2}$ are called reverse conflict, which is denoted as $x_{1} \# x_{2}$. In Figure 3(b), $c 2$ 
and $c 3$ are subjected to reverse conflict because $e 1 \leq c 2, e 2 \leq c 3$, and $e 1^{\bullet} \cap e 2^{\bullet}=\{c 1\} \neq \varnothing$. Similarly, reverse conflicts are found between $c 2$ and $c 4$ and between $e 1$ and $e 2$.

(3) Reverse concurrency: If $\neg\left(x_{1} \leq x_{2} \vee x_{2} \leq x_{1} \vee x_{1} \# x_{2}\right), x_{1}$ and $x_{2}$ are subjected to reverse concurrency, which is denoted as $x_{1} \| x_{2}$. In Figure 3(b), reverse concurrency is found between $c 3$ and $c 4$.

RON satisfies the following three properties.

(1) $\forall c \in C:\left|c^{\bullet}\right| \leq 1$;

(2) There is no reverse self-conflict in RON, namely that there is no event $e \in E$ making $e \# e$.

(3) $F^{\prime}$ is loop-free, namely that the reflexive and (irreflexive) transitive closure of $F^{\prime}$ is a partial order.

Definition 2 (Reverse configuration). The reverse configuration $C f g$ of $R O N$, which is a set of several events, satisfies the following two properties.

(1) If an event $e \in C f g$, then $\forall e^{\prime}<e: e^{\prime} \in C f g$;

(2) There are no events subjected to reverse conflict in $C f g$, namely $\forall e, e^{\prime} \in C f g: \neg\left(e \# e^{\prime}\right)$.

Definition 3 (Reverse local configuration). $\left\{e^{\prime} \mid e^{\prime} \in E \wedge e^{\prime}<e\right\}$ is defined as the reverse local configuration of the event $e$, which is denoted as $[e]$.

In Figure 3(b), $[e 1]=\{e 1\},[e 3]=\{e 2, e 3\}$.

Definition 4 (Reverse cut). For a configuration $C f g$, its reverse cut is defined as $C u t(C f g)=\left(C M_{f} \cup{ }^{\bullet} C f g\right) \backslash C f g^{\bullet}$. In addition, for a condition set, if any two elements are reversely concurrent, one of the two elements is called a co-set. It is not difficult to find that $\operatorname{Cut}(\mathrm{Cfg})$ is a co-set.

In Figure 3(b), $\operatorname{Cut}([e 2])=\{c 3, c 4\}, \operatorname{Cut}([e 3])=\{c 5\}$.

Reverse configuration and reverse cut are used to establish the mapping relationship of markings between the reverse occurrence net and the Petri net in Section 2.1.3.

\subsubsection{Reverse unfolding}

For a given Petri net $\Sigma=\left(P, T, F, M_{0}\right)$, the coverability of the target marking $M_{f}$ should be verified. The mapping relationship $\mu: C \cup E \rightarrow P \cup T$ between nodes in $\Sigma$ and $R O N=\left(C, E, F^{\prime}, C M_{f}\right)$ is defined as follows.

(1) If $c \in C$, then $\mu(c) \in P$; if $e \in E$, then $\mu(e) \in T$;

(2) $\forall e \in E, \bullet e$ to $\bullet \mu(e)$ satisfies the bijective relationship under the constraint of $\mu$, and $e^{\bullet}$ to $\mu(e)^{\bullet}$ satisfies the injective relationship under the constraint of $\mu$. Differently from forward unfolding, in reverse unfolding, $e^{\bullet}$ to $\mu(e)^{\bullet}$ may not satisfy the surjective relationship.

(3) $C M_{f}$ and $M_{f}$ satisfy the bijective relationship under the constraint of $\mu$.

Definition 5 (Reverse marking). $\operatorname{Mark}(C f g)=\mu(\operatorname{Cut}(C f g))$ is defined as the reverse marking of the configuration $C f g$.

$\operatorname{Mark}(C f g)$ can be viewed as an intermediate marking of $R O N$, and the coverability determination of $M_{f}$ can be converted to the coverability determination of $\operatorname{Mark}(C f g)^{[19]}$.

In Figure 3(b), $\operatorname{Mark}([e 2])=\mu(\{c 3, c 4\})=\{p 2, p 3\}, \operatorname{Mark}([e 3])=\mu(\{c 5\})=\{p 1\}$.

Definition 6 (Reverse unfolding). On the basis of the above concepts, the reverse unfolding of the target marking $M_{f}$ in the Petri net $\Sigma$ is defined as a 2-tuple $R U n f(R O N, \mu)$, which satisfies the following properties:

(1) RUnf is complete: Let the initial marking of $\Sigma$ be $M_{0}$. If $M_{0} \mapsto M_{f}$, there is a reverse configuration $C f g$ in $R U n f$ which satisfies $\operatorname{Mark}(C f g) \subseteq M_{0}$. Here we just need to make sure that the coverability of $M_{f}$ is not broken, and we do not need to obtain all triggered sequences. 
(2) RUnf is finite, namely that RUnf includes finite conditions and events.

\subsection{Reverse unfolding algorithm}

With a given Petri net $\Sigma=\left(P, T, F, M_{0}\right)$ and a given target marking $M_{f}$ whose coverability needs to be determined, the basic principle of the reverse unfolding algorithm is as follows. First, for each place of $M_{f}$, a corresponding condition is added to $R U n f$, that is, create $C M_{f}$. Specifically, after $C M_{f}$ is created, reverse extension is conducted from $C M_{f}$. The events that can generate these conditions as well as the conditions that enable these events are added, and the redundant events are cut off. The above steps are repeated until a reverse marking can be covered by the initial marking of $\Sigma$, or until the target marking is proved to be uncoverable.

Next, we provide the exact definitions of reverse extension and reverse cut-off events and introduce the approach of determining the coverability of target markings with the example shown in Figure 4. It is assumed that the target marking to be determined in Figure 4 is $\{p 6, p 7\}$.

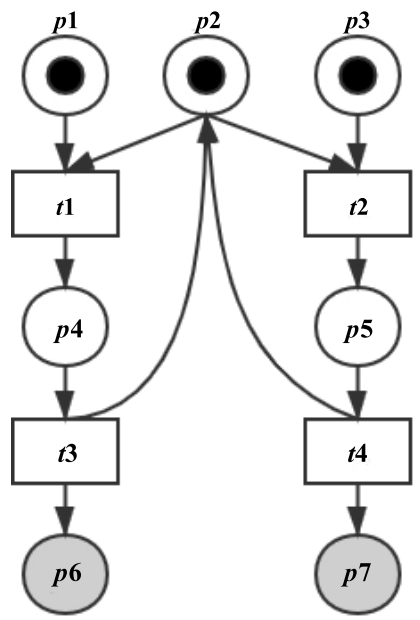

Figure 4 An example of Petri net

\subsubsection{Reverse extension}

Definition 7 (Reverse extension). A reverse extension is a 2-tuple rext $=(t, C)$, where $C$ is a co-set, and $\mu(C) \subseteq t^{\bullet}$. The set composed of reverse extensions is denoted as RExt.

For each reverse extension rext $=(t, C)$ selected from $R E x t$ and used to expand RUnf, an event $e=(t, C)$ needs to be added to $R U n f$. Meanwhile, a condition is added to RUnf for each place of ${ }^{\bullet} t$. Then RExt is recalculated. This process is denoted as $N E(R U n f, e)$.

In $N E(R U n f, e)$, the candidate extension set is calculated as follows. The introduction of - $e$ adds a new co-set for $R U n f$. For a newly added co-set $C$, if there is a transition $t$ satisfying $\mu(C) \subseteq t^{\bullet}$, then a new candidate extension rext $=(t, C)$ is added to $R E x t$.

According to the above definitions, there are massive redundant extensions in RExt. For example, if it is assumed that $\{c 1, c 2, c 3\}$ is a co-set, according to the definition of a co-set, $\{c 1, c 2\},\{c 1, c 3\},\{c 2, c 3\},\{c 1\},\{c 2\}$, and $\{c 3\}$ are all co-sets. If there is a reverse extension $(t,\{c 1, c 2, c 3\})$, there are reverse extensions $(t,\{c 1, c 2\}),(t,\{c 1, c 3\}),(t,\{c 2, c 3\}),(t,\{c 1\})$, $(t,\{c 2\})$, and $(t,\{c 3\})$. Thus, if there are no additional constraints, the scale of RExt will be huge. As a result, we add the two following conditions for RExt. 
(1) For an event $e$ in RUnf, there is no reverse extension rext $=(t, C)$ in $R E x t$ which makes $\mu(e)=t \wedge e^{\bullet}=C$;

(2) For two reverse extensions rext $t_{1}=\left(t_{1}, C_{1}\right)$ and rext $t_{2}=\left(t_{2}, C_{2}\right)$ in RExt, if $t_{1}=$ $t_{2} \wedge C_{1} \subset C_{2} \wedge \operatorname{Mark}\left(\left[\operatorname{rext}_{1}\right]\right) \geq \operatorname{Mark}\left(\left[\operatorname{rext}_{2}\right]\right)$, we delete the extension $\operatorname{rext}_{1}$ from $R E x t$. Here we assume $e$ is the corresponding event of rext in RUnf, and [rext] can be viewed as [e].

In condition (2), $\operatorname{Mark}\left(\left[\operatorname{rext}_{1}\right]\right) \geq \operatorname{Mark}\left(\left[\operatorname{rext}_{2}\right]\right)$ seems to be unnecessary. In fact, Parosh proposed the concept of reverse unfolding in Reference [19], and only used the constraint $t_{1}=t_{2} \wedge C_{1} \subset C_{2}$ in condition (2). However, this breaks the completeness of reverse unfolding and causes errors in some cases applying the reverse unfolding algorithm. Appendix $B$ provides the analysis of relevant counterexamples. For this reason, we add the constraint $\operatorname{Mark}\left(\left[\operatorname{rext}_{1}\right]\right) \geq \operatorname{Mark}\left(\left[\operatorname{rext}_{2}\right]\right)$. Many cases prove that it is effective.

In Figure 5, we use the reverse extension rext $=(t 1,\{c 3\})$ to generate the event $e 2$ and yield the precondition $\{c 4, c 5\}$ of $e 2$ at the same time. The addition of $\{c 4, c 5\}$ generates new cosets as well as the corresponding reverse extensions $(t 3,\{c 5\}),(t 4,\{c 5\})$, and $(t 4,\{c 2, c 5\})$. For $\operatorname{rext}_{1}=(t 4,\{c 5\})$ and $\operatorname{rext}_{2}=(t 4,\{c 2, c 5\})$, there is $\operatorname{Mark}\left(\left[\operatorname{rext}_{1}\right]\right)=\{p 1, p 5, p 7\} \geq$ $\{p 1, p 5\}=\operatorname{Mark}\left(\left[\operatorname{rext}_{2}\right]\right)$, and we delete rext 1 according to the condition (2) of RExt. We finally obtain $N E(R U n f, e)=\{(t 3,\{c 5\}),(t 4,\{c 2, c 5\})\}$.
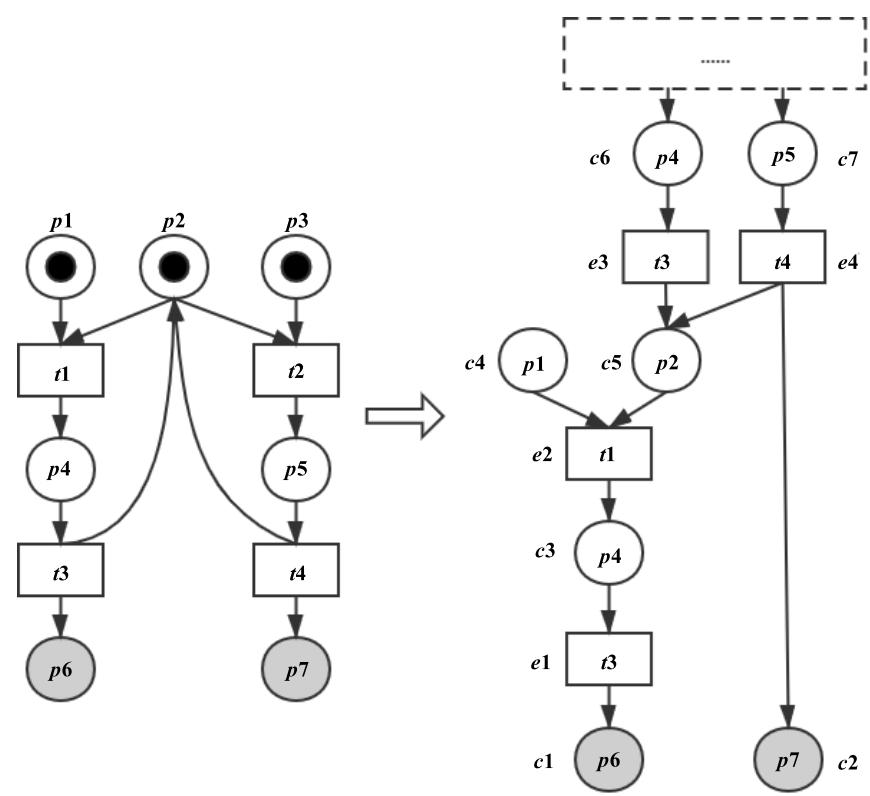

Figure 5 Reverse extension of Petri net shown in Figure 4 (partial)

\subsubsection{Reverse cut-off events}

The above reverse extension rule can assure the completeness of RUnf, but cannot guarantee its finiteness. Thus, the rule of the recognizing reverse cut-off events will be provided below to assure the terminability of the reverse unfolding processes.

Definition 8 (Reverse cut-off events). The event $e$ is a reverse cut-off event when and only when there is an event $e^{\prime}$ in RUnf satisfying the following two conditions.

(1) $\operatorname{Mark}\left(\left[e^{\prime}\right]\right) \leq \operatorname{Mark}([e])$.

(2) $\left[e^{\prime}\right] \prec[e]$. 
where $\prec$ is a partial order defined on configurations, which is called an adequate order. It satisfies the following three conditions.

(1) $\prec$ is well-founded.

(2) $\prec$ is a refinement of $\subset$, and $C f g_{1} \subset C f g_{2}$ means that $C f g_{1} \prec C f g_{2}$.

(3) If $\operatorname{Mark}\left(C f g_{1}\right) \leq \operatorname{Mark}\left(C f g_{2}\right)$ and $C f g_{1} \prec C f g_{2}$, for a prefix $E_{2}$ of $C f g_{2}$, there is $E_{1}$ satisfying $\operatorname{Mark}\left(C f g_{1} \oplus E_{1}\right) \leq \operatorname{Mark}\left(C f g_{2} \oplus E_{2}\right)$ and $C f g_{1} \oplus E_{1} \prec C f g_{2} \oplus E_{2}$. For a configuration $C f g, C f g \oplus E$ indicates that there is an event set $E$ satisfying $C f g \cap E=\varnothing$, where $C f g \cup E$ is also a configuration. $E$ is also called the prefix of $C f g$.

In this paper, we use $\prec_{r}$ as an adequate order, and Theorem 1 in Appendix A can be referred to for the relevant proof. $C f g_{1} \prec_{r} C f g_{2}$ is defined as

(1) $\left|C f g_{1}\right|<\left|C f g_{2}\right|$.

(2) $\left|C f g_{1}\right|=\left|C f g_{2}\right| \wedge \operatorname{Lex}\left(\mu\left(C f g_{1}\right)\right)<\operatorname{Lex}\left(\mu\left(C f g_{2}\right)\right)$, where $\mu(C f g)$ is a transition set of the configuration $\mathrm{Cfg}_{2}$ mapping to $\Sigma$, and it is a multiset. $\operatorname{Lex}(\mu(C f g))$ sorts the transitions in $\mu(C f g)$ by ID from small to large. This can be understood as when two configurations are of the same size, the lexicographical order of their corresponding transition sets is compared.

In Figure 5, the event $e 3$ is cut off due to the event $e 1$, as shown in Figure 6. This is because $|[e 1]|<|[e 3]|$, i.e., $[e 1] \prec_{r}[e 3]$, and $\operatorname{Mark}([e 1])=\{p 4, p 7\} \leq\{p 1, p 4, p 7\}=\operatorname{Mark}([e 3])$.

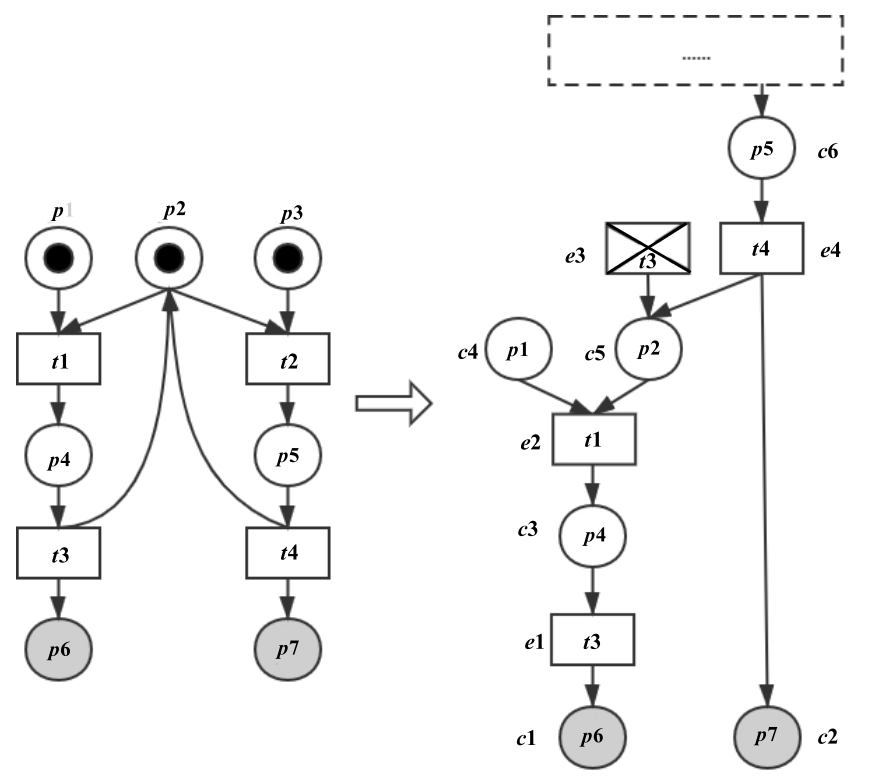

Figure 6 Cutting off event $e 3$ by event $e 1$

Reverse extension and reverse cut-off events guarantee the completeness and finiteness of $R U n f$, and theorems 2 and 3 in Appendix A can be referred to for the relevant proof.

\subsubsection{Coverability determination based on reverse unfolding}

In this paper, we only focus on 1-safe Petri nets, and we can add source place $p s$, source transition $t s$, and flow relation $(p s, t s)$ to Petri nets. Moreover, we add the flow relation $(t s, p)$ to each $p \in M_{0}$ to convert solving $M_{0} \mapsto M_{f}$ to solving $\left\{p_{s}\right\} \mapsto M_{f}$. This only needs to determine whether there is a configuration $C f g$ in $R U n f$ satisfying $\operatorname{Mark}(C f g)=\{p s\}$, after which the coverability of $M_{f}$ can be verified. With the Petri net in Figure 4 as an example, the Petri net after the addition of source nodes is as shown in Figure 7. 


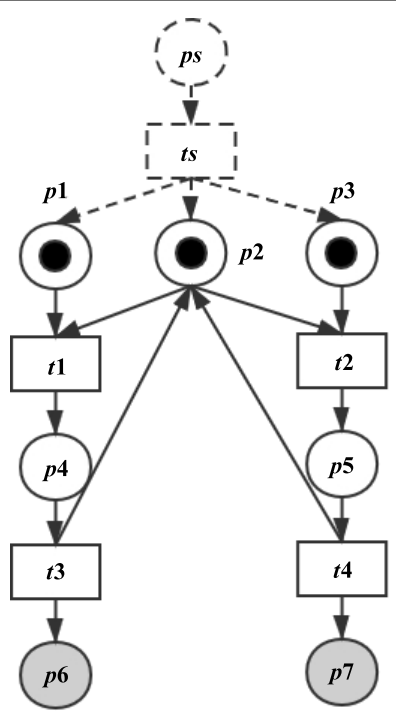

Figure 7 Petri net after source node addition

\subsubsection{Reverse unfolding algorithm and examples of coverability determination}

The flow of coverability determination of reverse unfolding can be summarized as follows. Initially, there is only the condition set $C M_{f}$ corresponding to $M_{f}$ in Runf, and the algorithm calculates the initial extension set RExt on the basis of $C M_{f}$. Subsequently, as long as RExt is not empty, the algorithm will continue to conduct reverse extension. In each extension, a $r e x t=(t, C)$ is randomly selected from $R E x t$, and a corresponding event $e=(t, C)$ is created in $R U n f$. If $e$ is not a reverse cut-off event, a relevant condition $c=(p, e)$ is created in $R U n f$ for each place $p$ in ${ }^{\bullet} t$. Then, we update $R E x t$ by calculating $R E x t=R E x t \cup N E(R U n f, e)$. If there is an event $e$ satisfying $\operatorname{Mark}([e])=\{p s\}$, it indicates that $M_{f}$ is coverable, and the algorithm ends. If $R E x t$ is empty finally and there is no event $e$ satisfying $\operatorname{Mark}([e])=\{p s\}$, it indicates $M_{f}$ is uncoverable. The pseudo-code of the reverse unfolding algorithm is as follows.

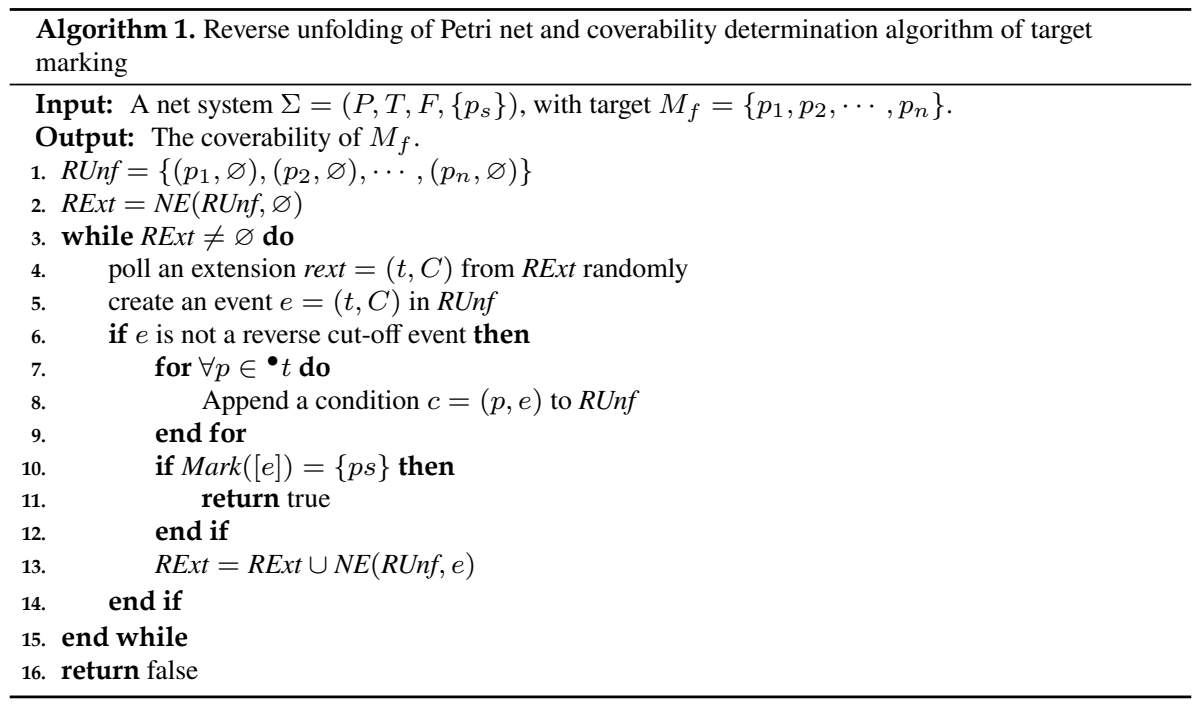


With the Petri net in Figure 7 as an example, the process of the coverability determination for the target marking $\{p 6, p 7\}$ by reverse unfolding is shown in Table 1.

Table 1 Example of reverse unfolding

\begin{tabular}{ll}
\hline & Process of reverse unfolding \\
\hline Step 0. & There are only conditions $c 1$ and \\
& $c 2$ in initial $R U n f$. \\
Step 1. & Select rext 1 to extend and create \\
& the event $e 1=(t 3,\{c 1\})$ and its \\
& precondition $\{c 3\}$ in $R U n f$.
\end{tabular}

Step 2. Select rext 3 to extend and create the event $e 2=(t 1,\{c 3\})$ and its precondition $\{c 4, c 5\}$ in $R U n f$ to generate new extensions $(t s,\{c 4\}),(t 3,\{c 5\})$, $(t 4,\{c 5\}),(t 4,\{c 2, c 5\})$. Delete the extension $(t 4,\{c 5\})$ according to the constraints in RExt.

Step 3. Select rext4 to extend and create the event $e 3=(t s,\{c 4\})$ and its precondition $\{c 6\}$ in RUnf. There is no new extension at this point.

Step 4. Select rext5 to extend and create the event $e 4=(t 3,\{c 5\})$ in RUnf. According to the definition of reverse cut-off events, $e 4$ will be cut off due to $e 1$, and this extension ends.

Step 5. Select rext6 to extend and create the event $e 5=(t 4,\{c 2, c 5\})$ and its precondition $\{c 7\}$ in $R U n f$.

Step 6. Select rext7 to extend and create the event $e 6=(t 2,\{c 7\})$ and its precondition $\{c 8, c 9\}$ in $R U n f$ to generate new extensions

$$
\begin{aligned}
& \text { RExt } \\
& \{\text { rext } 1=(t 3,\{c 1\}), \\
& \text { rext } 2=(t 4,\{c 2\})\} \\
& \{\text { rext } 2=(t 4,\{c 2\}), \\
& \text { rext } 3=(t 1,\{c 3\})\} \\
& \{\text { rext } 2=(t 4,\{c 2\}), \\
& \text { rext } 4=(t s,\{c 4\}), \\
& \text { rext } 5=(t 3,\{c 5\}), \\
& \text { rext } 6=(t 4,\{c 2, c 5\})\}
\end{aligned}
$$

$\{$ rext $2=(t 4,\{c 2\})$, rext $5=(t 3,\{c 5\})$, rext $6=(t 4,\{c 2, c 5\})\}$

$\{$ rext $2=(t 4,\{c 2\})$, rext $6=(t 4,\{c 2, c 5\})\}$

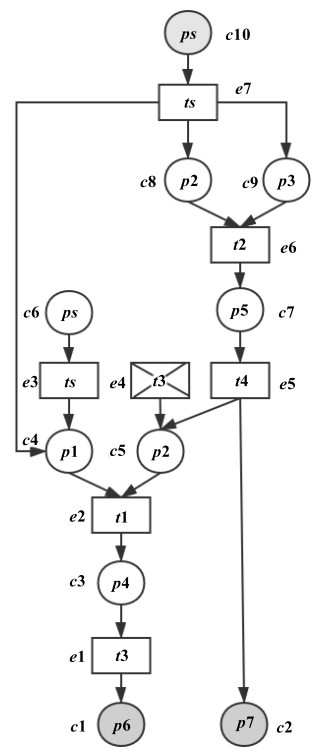
$(t s,\{c 8\}),(t s,\{c 9\}),(t s,\{c 8$, $c 9\}),(t s,\{c 4, c 8\}),(t s,\{c 4$, $c 9\}),(t s,\{c 4, c 8, c 9\})$, $(t 3,\{c 8\})$, and $(t 4,\{c 8\})$. Only reserve extensions (ts, $\{c 4, c 8$, $c 9\}),(t 3,\{c 8\})$, and $(t 4,\{c 8\})$ are reserved according to the constraints of RExt.

Step 7. Select rext 8 to extend and create the event $e 7=(t s,\{c 4, c 8, c 9\})$ and its precondition $\{c 10\}$ in RUnf. The target marking is coverable at this point, and the algorithm ends.

$\{$ rext $2=(t 4,\{c 2\})$, rext $7=(t 2,\{c 7\})\}$

$\{$ rext $2=(t 4,\{c 2\})$, rext $8=$

$(t s,\{c 4, c 8, c 9\})$, rext $9=(t 3,\{c 8\})$, rext $10=(t 4,\{c 8\})\}$

RUnf




\subsection{Heuristic optimization of reverse unfolding algorithm}

The extension sequence has a critical impact on the efficiency of coverability determination of target markings. For this purpose, this paper proposes three heuristic techniques based on practices by referring to Reference [17].

(1) The block strategy.

In the reverse extension rext $=(t, C), C$ and $t^{\bullet}$ may do not satisfy a surjective relationship. However, practices show that when the relationship between $C$ and $t^{\bullet}$ is not surjective, it often means that rext is generated too early, and the corresponding conditions have not been yielded. At this point, the extensions guided by it will be redundant. To avoid this situation as far as possible, we add the reverse extensions that do not satisfy the surjective relationship to the blocking queue and select the reverse extensions that satisfy the surjective relationship with priority. Only when all reverse extensions do not satisfy the surjective relationship, a reverse extension is selected from the blocking queue for activation. In practice, the block strategy is usually used together with other heuristic strategies.

(2) The hmax strategy.

The hmax strategy is a distance-based heuristic strategy proposed by Bonet in Reference [17]. $d\left(M, M^{\prime}\right)$ is defined as a distance between markings $M$ and $M^{\prime}$. In the hmax strategy, the distance between $M$ and the transition $t$ is defined as $\max _{p \in t} d(M,\{p\})$, and the distance between $M$ and the place $p$ is defined as $1+\min _{t \in p} d(M, t)$, and so on. When calculating $d\left(M, M^{\prime}\right)$, we only need to solve the maximum distance between $M$ and each place in $M^{\prime}$, namely to calculate $\max _{p \in M^{\prime}} d(M,\{p\})$.

The above process can be summarized as

$$
d\left(M, M^{\prime}\right)= \begin{cases}0, & M^{\prime} \subseteq M \\ 1+\min _{t \in p} d(M, t), & M^{\prime}=\{p\} \\ \max _{p \in M^{\prime}} d(M,\{p\}), & \text { otherwise }\end{cases}
$$

(3) The hsum strategy.

hsum is very similar to hmax. The difference is that hsum defines the distance between $M$ and the transition $t$ as $\sum_{p \in t} d(M,\{p\})$. Correspondingly, when calculating $d\left(M, M^{\prime}\right)$, we sum up the distances between $M$ and each place in $M^{\prime}$, namely calculate $\sum_{p \in M^{\prime \prime}} d(M,\{p\})$.

The above process can be summarized as

$$
d\left(M, M^{\prime}\right)= \begin{cases}0, & M^{\prime} \subseteq M \\ 1+\min _{t \in p} d(M, t), & M^{\prime}=\{p\} \\ \sum_{p \in t} d(M,\{p\}), & \text { otherwise }\end{cases}
$$

In practice, we will take into account the size of configurations when using the hmax and hsum strategies. Specifically, for two reverse extensions rext $t_{1}=\left(t_{1}, C_{1}\right)$ and rext $t_{2}=\left(t_{2}, C_{2}\right)$, if $\left|\left[\operatorname{rext}_{1}\right]\right|+d\left(M_{0}, \operatorname{Mark}\left(\left[\operatorname{rext}_{1}\right]\right)\right)<\left|\left[\operatorname{rext}_{2}\right]\right|+d\left(M_{0}, \operatorname{Mark}\left(\left[\operatorname{rext}_{2}\right]\right)\right)$, we will select $\operatorname{rext}_{1}$ with priority for extension.

\section{Data Race Detection Based on Reverse Unfolding}

Due to the uncertainty of thread scheduling, multithreaded programs are often accompanied by data race. Data race means that multiple threads access the same memory address space in a non-thread-safe situation. It can affect program results and even lead to system crashes. Since data race usually occurs only in some specific thread traces, it poses a great challenge 
for developers to detect data race. Several serious incidents in history, such as the radiation therapy machine Therac- 25 accident ${ }^{[20]}$, the massive blackout in North America in $2003^{[21]}$, and the FaceBook failure in NASDAQ ${ }^{[22]}$, were all related to data race. Data race detection is mainly divided into two categories: static detection ${ }^{[23-25]}$ and dynamic detection ${ }^{[26-28]}$. In this section, we conduct the static detection of data race in Java concurrent programs based on reverse unfolding of Petri nets.

\subsection{Building the Petri net model for programs}

Krishna ${ }^{[29]}$ built Petri net models for synchronization primitive and flow control statements of C-Pthread programs. In this paper, we apply this modelling method to Java concurrent programs. The models of the following four kinds of statements are built.

(1) Thread starting and merging: In Java, the starting and merging of the thread $t$ correspond to $t . s t a r t()$ and $t$.join (). When t.start() is invoked, the state of the thread $t$ changes to Runnable. After the CPU scheduling is obtained, the thread $t$ runs formally in the Running state. After t.join () is invoked, the state of the current thread changes to Blocked, and changes to Runnable until the thread $t$ is executed. Then it re-waits the CPU scheduling. The corresponding Petri net model of thread starting and merging is shown in Figure 8(a).

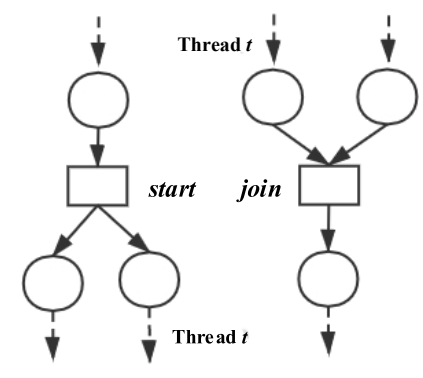

(a) Thread starting and merging

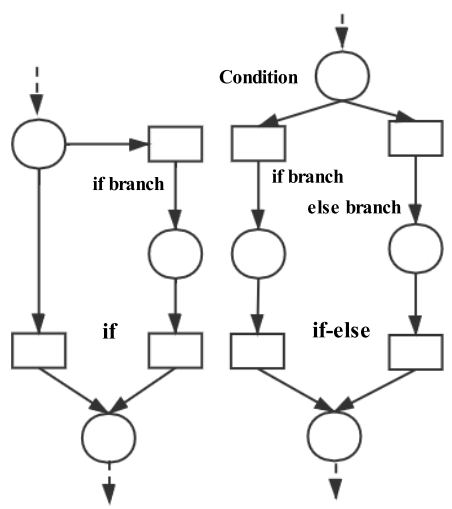

(c) Branches in programs

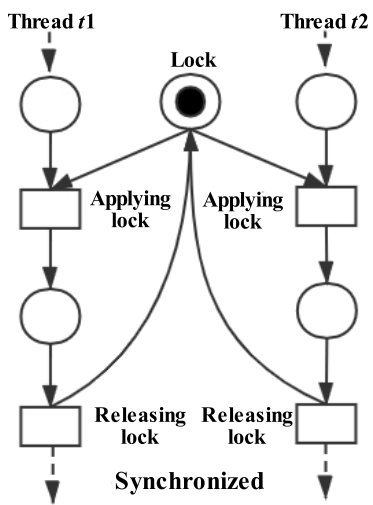

(b) Lock application and release

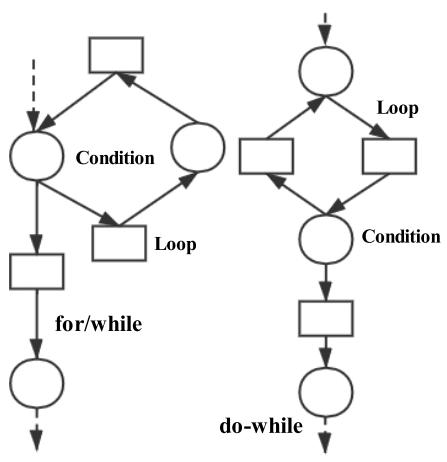

(d) Loops in programs

Figure 8 Petri net models of Java concurrent programs

(2) Lock application and release: Java uses synchronized statements to achieve mutual 
exclusion of threads, including synchronized methods and synchronized code blocks, which are essentially the same. When the thread $t 1$ and the thread $t 2$ access the synchronized code block at the same time, only one thread can get access. The Petri net model of lock application and release is shown in Figure 8(b).

(3) Branches: Branches in Java are equivalent to if and if-else branches logically. The corresponding Petri net model is shown in Figure 8(c).

(4) Loops: Loops in Java programs are divided into for/while loops and do-while loops. The two have similar structures, and they both consist of a control condition and a loop body. The corresponding Petri net model is shown in Figure 8(d).

Figure 9 shows an example of Java multithreaded program and its Petri net model constructed in line with the above rules. The transition with an asterisk corresponds to a program statement, and other transitions are merely used for indicating program structure. Specifically,

(1) For the program statements, $t 1$ indicates creating the thread $t 1 ; t 6$ indicates creating the thread $t 2 ; t 14$ indicates merging the thread $t 1$ to the main thread; $t 7$ and $t 8$ indicate applying the lock; $t 15$ and $t 16$ indicates releasing the lock; $t 9$ corresponds to the statement $x=2 ; t 10$ corresponds to the statement $x=1 ; t 11$ corresponds to the statement System.out.println $(x)$.

(2) In terms of program structure, $t 2$ and $t 4$ indicates the entry into the if structure; $t 13$ and $t 17$ indicates the exit from the if structure; $t 3$ indicates the beginning of a loop; $t 5$ indicates the end of a loop; $t 12$ indicates the exit from a loop.

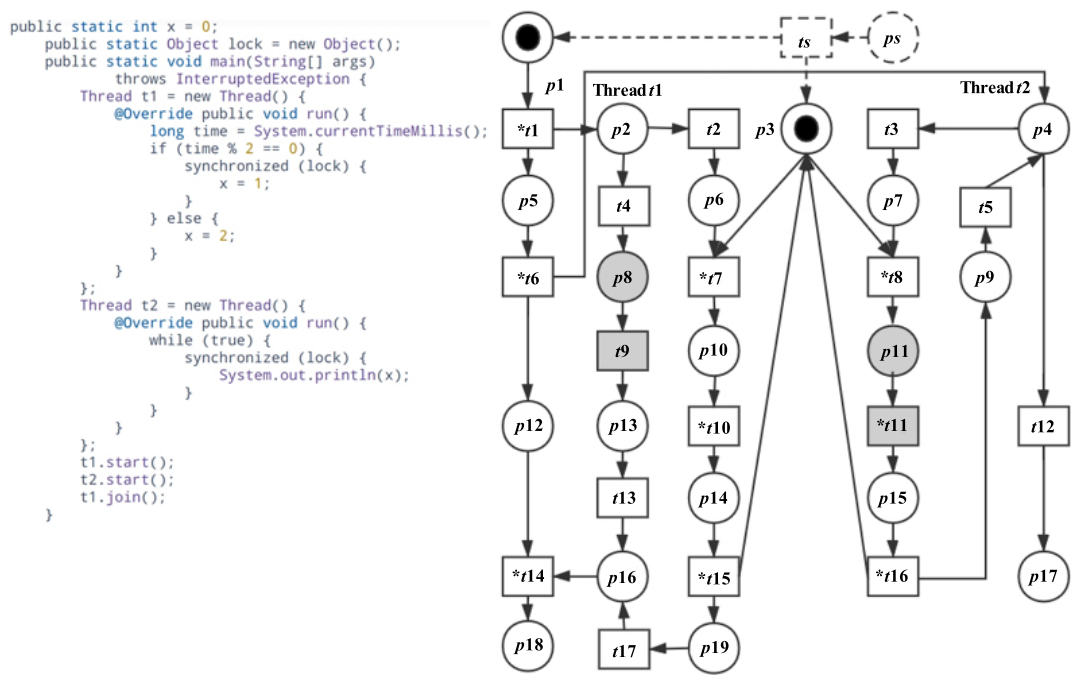

Figure 9 Java source code (left) and its Petri net model (right)

\subsection{Data race detection based on coverability determination}

Two read/write or write/write operations on a same shared variable in a program do not result in data race if they have sequential dependencies in the execution order. On the contrary, if they are concurrent, it will lead to data race. In view of this, we can analyze the Petri net model of the program to determine whether the transitions corresponding to the two operations have the concurrent relationship in some states. In fact, for any two transitions $t$ and $t^{\prime}$, we only need to determine whether there is a reachable marking $M$ satisfying $M \geq{ }^{\bullet} t+{ }^{\bullet} t^{\prime}$, namely to determine the coverability of the marking ${ }^{\bullet} t+{ }^{\bullet} t^{\prime}$.

For the example shown in Figure 9, data race occurs if the operation of reading the shared variable System.out.println $(x)$ and the operation of writing the shared variable $x=2$ do not 
have a same lock. The write operation $x=2$ of the shared variable $x$ corresponds to the transition $t 9$, and the read operation System.out.println $(x)$ corresponds to the transition $t 11$. To determine data race, we only need to verify the coverability of the target marking $\{p 8, p 11\}$.

The reverse unfolding flow of the Petri net model of the program is shown in Table 2.

Table 2 Example of data race detection for concurrent programs

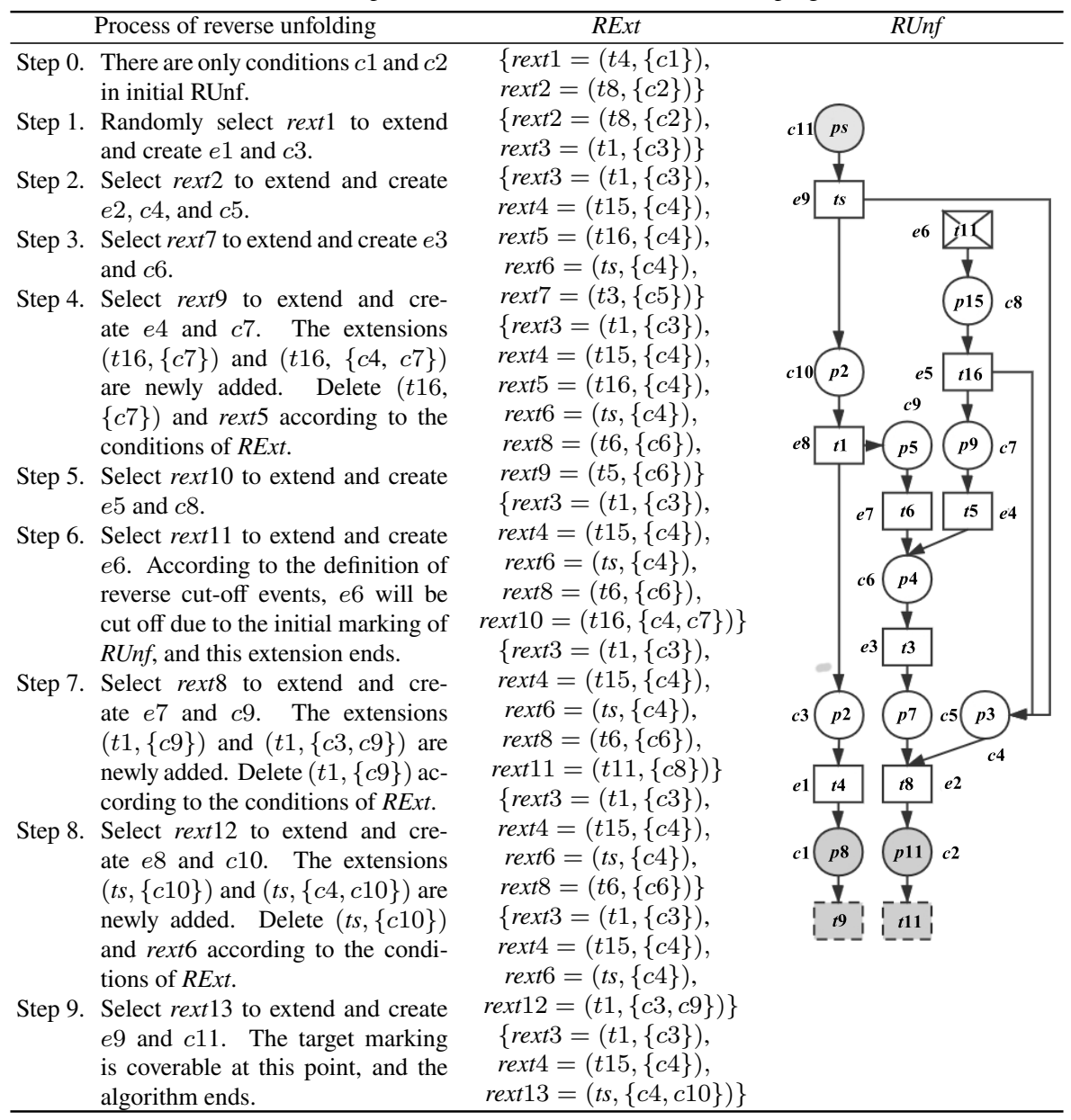

It is not difficult to find that $\operatorname{Mark}([e 9])=\{p s\}$. Thus the target marking $\{p 8, p 11\}$ is coverable. The transitions corresponding to the read and write operations can be concurrent, and the program has potential data race.

In this section, we build the Petri net model of a Java concurrent program and transform the data race detection to the coverability verification of relevant transitions. With an example, we show the application of the reverse unfolding algorithm in data race detection. However, this paper only provides the Petri net modelling method for Java multithreaded programs, but does not design a modelling tool. In practice, Soot ${ }^{[30]}$ can be used for the automatic modelling of Java concurrent programs. Soot is a Java bytecode optimization framework, by which the Java source code can be converted into Jimple intermediate code for analysis. For example, we can use JInvokeStmt to analyze the starting and merging of threads and to invoke many functions; we can adopt JEnterMonitorStmt and JExitMonitorStmt to analyze lock application and release; 
we can leverage JIfStmt and JGotoStmt to analyze branches and loops in programs. Limited by space, we will study this in our future work.

\section{Experimental Evaluation}

In this section, we compare the efficiency of heuristic reverse unfolding and directed unfolding ${ }^{[17]}$ (a forward unfolding also using heuristics) on the coverability problem of Petri nets. In this paper, the algorithm efficiency is evaluated by the total number of events and operation time. The reference library used in the experiment contains 415 test cases, including 358 cases of directed unfolding and 57 cases constructed by us. All of them are coverable. The number of transitions of Petri nets varies from 6 to 9,462, and there are 261,694 transitions in total. The experiment is conducted on a machine equipped with AMD Ryzen 7 4800U and 16 GB RAM. The source code and test cases can be acquired from Reference [31].

\subsection{Comparison between reverse unfolding and forward unfolding without heuristic strategy}

As the heuristic strategy has a great impact on the algorithm efficiency, it is unfavorable to visualize the property advantages of reverse unfolding. Thus, this paper first uses the basic breadth-first and depth-first strategies to compare the two algorithms (Reference [18] can be referred to for the forward unfolding). The case set randomtree in the experiment constructs 37 Petri nets in a tree structure. Each node in the tree is a cyclic model similar to that in Figure 8 (d). We conduct coverability determination by randomly selecting markings of leaf nodes. The maximum number of extension times of algorithms is set as 10,000 , and the maximum operation time is $35 \mathrm{~s}$. The experimental results are shown in Table 3.

The results show that the reverse unfolding based on the depth-first strategy performs best in randomtree. The scale of $|E|$ is optimal on all 37 cases. Meanwhile, the efficiency of reverse unfolding is higher than that of forward unfolding no matter depth-first or breadth-first strategies are used. This is because randomtree has a tree structure. The forward unfolding implicitly portrays the complete behavior of the system and covers all the branches of the tree structure. In contrast, the reverse unfolding only describes the system states related to coverability determination and covers only a branch of the tree.

\subsection{Comparison of reverse unfolding and forward unfolding in the presence of heuristic strategies}

In this section, we compare the efficiencies of heuristic reverse unfolding and directed unfolding in the coverability problem of Petri nets. In realizing directed unfolding, we use the heuristic strategies hmax and hsum in Reference [17] and the property that the extension sequence can be separated from the partial order of cut-off events in Reference [18]. In implementing reverse unfolding, we use the heuristic strategies block, hmax, and hsum stated in Section 2.3. In addition, implementation details of the two methods are kept consistent as far as possible. For comparison purposes, we only select the optimal heuristic strategy in directed unfolding and reverse unfolding for each group of experiments. The relevant experiments are as follows.

(1) randomtree

In Section 4.1, we only compare the efficiencies of forward unfolding and reverse unfolding without heuristics. Herein, we compare them under the heuristic technology. The maximum number of extension times is set as 10,000, and the maximum operation time is $35 \mathrm{~s}$. The experimental results are shown in Table 4 and Figure 10(a).

The results show that the reverse unfolding based on the block + depth-first strategy performs the best in randomtree. The scale of $|E|$ is optimal in all 37 cases. It should be noted that the 
efficiency of the forward unfolding combined with the hmax strategy is improved significantly compared with that without the heuristic strategy. This indicates, to some extent, the heuristic technique can compensate for property deficiencies of algorithms.

Table 3 Results of forward unfolding and reverse unfolding in randomtree without heuristic strategy

\begin{tabular}{|c|c|c|c|c|c|c|c|c|c|}
\hline \multirow{2}{*}{ Test case } & \multirow{2}{*}{$|T|$} & \multicolumn{2}{|c|}{ Unf-bfs } & \multicolumn{2}{|c|}{$U n f-d f s$} & \multicolumn{2}{|c|}{$R U n f-b f s$} & \multicolumn{2}{|c|}{$R U n f-d f s$} \\
\hline & & $|E|$ & Time $(\mathrm{ms})$ & $|E|$ & Time $(\mathrm{ms})$ & $|E|$ & Time $(\mathrm{ms})$ & $|E|$ & Time $(\mathrm{ms})$ \\
\hline randomtree100 & 899 & 500 & 64 & 448 & 125 & 36 & 34 & 29 & 42 \\
\hline randomtree 125 & 1,194 & 318 & 20 & 1,291 & 309 & 23 & 25 & 18 & 15 \\
\hline randomtree 150 & 1,387 & 656 & 98 & 428 & 21 & 38 & 15 & 29 & 18 \\
\hline randomtree 175 & 1,729 & 1,165 & 241 & 1,082 & 115 & 42 & 14 & 34 & 17 \\
\hline randomtree200 & 1,854 & 886 & 60 & 1,721 & 197 & 36 & 5 & 27 & 12 \\
\hline randomtree 225 & 2,130 & 134 & 3 & 4,640 & 2,575 & 15 & 3 & 12 & 2 \\
\hline randomtree 250 & 2,429 & 1,278 & 106 & 1,125 & 96 & 34 & 44 & 26 & 4 \\
\hline randomtree 275 & 2,575 & 1,390 & 148 & 2,309 & 415 & 43 & 8 & 34 & 5 \\
\hline randomtree 300 & 2,898 & 1,690 & 248 & 1,231 & 132 & 46 & 7 & 37 & 23 \\
\hline randomtree 325 & 3,132 & 1,359 & 143 & 1,238 & 129 & 33 & 22 & 28 & 4 \\
\hline randomtree 350 & 3,492 & 2,128 & 330 & 383 & 20 & 40 & 12 & 31 & 8 \\
\hline randomtree 375 & 3,615 & 2,134 & 404 & 2,082 & 361 & 39 & 5 & 31 & 5 \\
\hline randomtree 400 & 3,712 & 1,658 & 206 & 5,942 & 7,139 & 37 & 4 & 31 & 4 \\
\hline randomtree 425 & 4,076 & 1,166 & 91 & 9,642 & 20,789 & 38 & 3 & 29 & 4 \\
\hline randomtree 450 & 4,304 & 2,030 & 296 & 2,600 & 611 & 45 & 10 & 33 & 17 \\
\hline randomtree 475 & 4,523 & 613 & 30 & 4,801 & 2,654 & 25 & 4 & 20 & 2 \\
\hline randomtree500 & 4,732 & 1,476 & 141 & 2,887 & 887 & 34 & 24 & 27 & 5 \\
\hline randomtree525 & 5,014 & 1,828 & 204 & 5,781 & 6,730 & 37 & 4 & 30 & 4 \\
\hline randomtree 550 & 5,160 & 2,059 & 308 & 159 & 4 & 38 & 3 & 30 & 18 \\
\hline randomtree 575 & 5,394 & 1,008 & 77 & 8,720 & 13,284 & 36 & 5 & 27 & 14 \\
\hline randomtree 600 & 5,739 & 2,610 & 483 & - & - & 42 & 7 & 32 & 18 \\
\hline randomtree625 & 5,835 & 1,314 & 123 & 891 & 70 & 29 & 4 & 24 & 7 \\
\hline randomtree 650 & 6,238 & 2,760 & 651 & 9,494 & 24,708 & 43 & 4 & 31 & 4 \\
\hline randomtree 675 & 6,423 & 2,829 & 630 & 6,615 & 10,874 & 41 & 4 & 31 & 5 \\
\hline randomtree700 & 6,529 & 1,427 & 133 & 6,257 & 8,508 & 34 & 4 & 26 & 5 \\
\hline randomtree725 & 7,026 & 4,759 & 3,183 & - & - & 63 & 8 & 51 & 10 \\
\hline randomtree 750 & 7,133 & 2,853 & 688 & 2,061 & 280 & 52 & 9 & 38 & 7 \\
\hline randomtree 775 & 7,415 & 5,005 & 5,516 & 2,340 & 525 & 63 & 12 & 49 & 9 \\
\hline randomtree 800 & 7,673 & 294 & 12 & 232 & 7 & 24 & 16 & 20 & 3 \\
\hline randomtree825 & 7,689 & 2,557 & 665 & - & - & 37 & 7 & 29 & 6 \\
\hline randomtree 850 & 8,030 & 267 & 10 & 5,549 & 6,056 & 21 & 3 & 15 & 5 \\
\hline randomtree 875 & 8,224 & 2,182 & 373 & - & - & 34 & 4 & 25 & 6 \\
\hline randomtree 900 & 8,402 & 3,507 & 1,247 & 9,323 & 20,989 & 34 & 5 & 28 & 8 \\
\hline randomtree 925 & 8,762 & 5,305 & 4,789 & 2,551 & 442 & 60 & 8 & 47 & 10 \\
\hline randomtree 950 & 9,135 & 6,247 & 7,353 & - & - & 75 & 10 & 59 & 12 \\
\hline randomtree 975 & 9,491 & 6,274 & 8,860 & 5,583 & 6,217 & 72 & 10 & 52 & 11 \\
\hline randomtree 1000 & 9,462 & 6,025 & 8,618 & 7,241 & 12,960 & 59 & 7 & 43 & 9 \\
\hline
\end{tabular}

(2) threadlock

The case set threadlock simulates a thread-lock model according to the following rules. It is assumed that there are $x$ threads and $y$ locks. The $x$ threads successively apply lock 1 , lock $2, \cdots$, lock $y$, and then successively release lock $y$, lock $y-1, \cdots$, lock 1 . The target marking is composed of the final places of these threads. The maximum number of extension times is set as 10,000, and the maximum operation time is $70 \mathrm{~s}$. The experimental results are shown in Table 5 and Figure 10(b).

The results show that the forward unfolding based on the depth-first strategy performs the best in threadlock. Among 20 cases, the scale of $|E|$ is the optimal in 15 cases and is similar to that of the reverse unfolding based on the block + depth-first strategy in other 5 cases. In addition, with the increase in the number of cases, the operation time of forward unfolding is 
much superior to that of reverse unfolding. However, in threadlock, the number of forward branches of Petri nets is similar to that of reverse branches, which makes their efficiency close in theory. However, this is not consistent with the experimental results. The further analysis demonstrates that as the number of cases rises, the number of redundant extensions in RExt increases rapidly, resulting in a slow operation speed of reverse unfolding. Additionally, a huge extension set is harmful for heuristics to make the correct selection. Thus, how to effectively reduce the number of redundant extensions is a key factor to improve the efficiency of reverse unfolding.

Table 4 Results of directed unfolding and reverse unfolding in randomtree

\begin{tabular}{ccccccc}
\hline \multirow{2}{*}{ Test case } & \multirow{2}{*}{$|T|$} & \multicolumn{2}{c}{ Unf-hmax } & & \multicolumn{2}{c}{ RUnf-blcok+dfs } \\
\cline { 3 - 4 } & & $|E|$ & Time $(\mathrm{ms})$ & & $|E|$ & Time $(\mathrm{ms})$ \\
\hline randomtree100 & 899 & 30 & 55 & & $\mathbf{2 9}$ & 40 \\
randomtree125 & 1,194 & 19 & 12 & $\mathbf{1 8}$ & 14 \\
randomtree150 & 1,387 & 32 & 21 & & $\mathbf{2 9}$ & 11 \\
randomtree175 & 1,729 & 38 & 32 & & $\mathbf{3 4}$ & 18 \\
randomtree200 & 1,854 & 31 & 38 & & $\mathbf{2 7}$ & 6 \\
randomtree225 & 2,130 & 13 & 23 & & $\mathbf{1 2}$ & 29 \\
randomtree250 & 2,429 & 28 & 47 & & $\mathbf{2 6}$ & 5 \\
randomtree275 & 2,575 & 36 & 49 & & $\mathbf{3 4}$ & 7 \\
randomtree300 & 2,898 & 39 & 32 & & $\mathbf{3 7}$ & 5 \\
randomtree325 & 3,132 & 29 & 29 & & $\mathbf{2 8}$ & 4 \\
randomtree350 & 3,492 & 34 & 45 & & $\mathbf{3 1}$ & 5 \\
randomtree375 & 3,615 & 35 & 45 & & $\mathbf{3 1}$ & 4 \\
randomtree400 & 3,712 & 33 & 28 & & $\mathbf{3 1}$ & 4 \\
randomtree425 & 4,076 & 32 & 34 & & $\mathbf{2 9}$ & 15 \\
randomtree450 & 4,304 & 34 & 55 & & $\mathbf{3 3}$ & 44 \\
randomtree475 & 4,523 & 22 & 37 & & $\mathbf{2 0}$ & 15 \\
randomtree500 & 4,732 & 29 & 85 & & $\mathbf{2 7}$ & 6 \\
randomtree525 & 5,014 & 32 & 90 & & $\mathbf{3 0}$ & 3 \\
randomtree550 & 5,160 & 33 & 133 & $\mathbf{3 0}$ & 4 \\
randomtree575 & 5,394 & 31 & 150 & $\mathbf{2 7}$ & 3 \\
\hline
\end{tabular}

Table 5 Results of directed unfolding and reverse unfolding in threadlock

\begin{tabular}{|c|c|c|c|c|c|c|c|}
\hline \multirow{2}{*}{ Test case } & \multirow{2}{*}{$|T|$} & \multicolumn{3}{|c|}{ Unf-dfs } & \multicolumn{3}{|c|}{ RUnf-blcok $+d f s$} \\
\hline & & $|E|$ & Time (ms) & $|E x t|$ & $|E|$ & Event (ms) & $|R E x t|$ \\
\hline threadlock2_1 & 6 & 8 & 20 & 1 & 8 & 10 & 5 \\
\hline threadlock 3 & 10 & 12 & 7 & 3 & 12 & 8 & 11 \\
\hline threadlock3_2 & 16 & 18 & 27 & 3 & 21 & 14 & 23 \\
\hline threadlock4_1 & 14 & 16 & 19 & 6 & 16 & 10 & 19 \\
\hline threadlock 42 & 22 & 24 & 7 & 6 & 28 & 8 & 41 \\
\hline threadlock4_3 & 30 & 32 & 8 & 6 & 40 & 7 & 60 \\
\hline threadlock5_1 & 18 & 20 & 9 & 10 & 20 & 2 & 29 \\
\hline threadlock5_2 & 28 & 30 & 5 & 10 & 35 & 5 & 62 \\
\hline threadlock5_3 & 38 & 40 & 8 & 10 & 50 & 7 & 91 \\
\hline threadlock6_1 & 22 & 24 & 5 & 15 & 24 & 3 & 43 \\
\hline threadlock6_2 & 34 & 36 & 20 & 15 & 42 & 5 & 84 \\
\hline threadlock6_3 & 46 & 48 & 23 & 15 & 60 & 25 & 129 \\
\hline threadlock8_4 & 78 & 80 & 29 & 28 & 104 & 39 & 291 \\
\hline threadlock10_5 & 118 & 120 & 34 & 45 & 160 & 87 & 554 \\
\hline threadlock12_6 & 166 & 168 & 87 & 66 & 228 & 105 & 943 \\
\hline threadlock14_7 & 222 & 224 & 85 & 91 & 308 & 223 & 1,474 \\
\hline threadlock16_8 & 286 & 288 & 72 & 120 & 400 & 161 & 2,186 \\
\hline threadlock18_9 & 358 & 360 & 70 & 153 & 504 & 399 & 3,088 \\
\hline threadlock20_10 & 438 & 440 & 131 & 190 & 620 & 497 & 4,211 \\
\hline threadlock50_25 & 2,598 & 2,600 & 3,746 & 1,225 & 3,800 & 49,214 & 63,785 \\
\hline
\end{tabular}


(3) Reference libraries of directed unfolding

The directed unfolding uses four case sets, i.e., dartes, random, airport, and openstacks. We use them to perform experiments. The maximum number of extension times of the four use case sets is selected as 10,000, and the maximum operation time is $35 \mathrm{~s}$.

The experimental results of the case set dartes are shown in Figure 10(c). In forward unfolding, the hsum strategy performs the best, and the coverability of 256/257 cases is verified. In reverse unfolding, the hmax strategy has the best effect, which verifies the coverability of $257 / 257$ cases. Further, the scale of $|E|$ in reverse unfolding achieves the best effect in 48 cases and is comparable to that of forward unfolding in 2 cases. In this case set, forward unfolding and reverse unfolding both develop their own advantages.

In the case set random, the forward unfolding combined with the hsum strategy performs the best, and the coverability of 33/45 use cases is verified. In reverse unfolding, the block + hsum strategy is outstanding, which verifies the coverability of $26 / 45$ cases. Further, the scale of $|E|$ in forward unfolding performs the best in 33 cases and is comparable to that of reverse unfolding in 12 use cases. In the subsequent tests on airport and openstacks, the forward unfolding combined with the hsum strategy verifies the coverability of 19/26 and 30/30 cases, respectively, while reverse unfolding cannot verify the coverability of any cases. The low efficiency or even failure regarding these cases indicates that reverse unfolding is not applicable to all scenarios, which is determined by its property. However, the heuristic technique can compensate for deficiencies of algorithms in properties. Thus, we should design more effective heuristics to improve the low efficiency or even failure of reverse unfolding.

In this section, we compare the efficiencies of heuristic reverse unfolding and directed unfolding in coverability determination of Petri nets. The results show that in 415 groups of test data, the scale of reverse unfolding is better than that of forward unfolding for 85 data groups and is comparable to that of forward unfolding for 26 data groups. The experiment verifies that reverse unfolding is effective in some cases. If a Petri net has many forward branches, reverse unfolding can start from the target marking and only describes the system states related to coverability determination, so that the efficiency of coverability determination of target markings can be improved. However, reverse unfolding still has a low efficiency in most cases currently due to the following two reasons. The first is the number of redundant extensions. Too many redundant extensions result in slow operation of the algorithm and the heuristic cannot help to make correct selections. The second is the algorithm properties. When there are too many reverse branches in a Petri net, the efficiency of reverse unfolding is always lower than that of forward unfolding. However, the heuristic technique can compensate for property deficiencies of algorithms. Thus, we should design more effective heuristics to improve the low efficiency or failure of reverse unfolding.

\section{Conclusion and Prospect}

This paper proposed a target-oriented reverse unfolding algorithm for the coverability determination of safe Petri nets. Starting from the target marking that needs coverability determination, reverse unfolding only describes the system states related to coverability determination. In addition, it reduces the unfolding scale with heuristic techniques such as block, hmax, and hsum strategies, so as to improve the efficiency of coverability determination of target markings. Further, we apply the reverse unfolding algorithm to formal verification of concurrent programs and convert data race detection of concurrent programs to coverability determination of specific markings of Petri nets. We compare the efficiencies of heuristic reverse unfolding and directed unfolding in coverability of Petri nets by experiments. It is verified that reverse unfolding can improve the coverability determination efficiency when Petri nets have many forward branches. 
However, this paper still has some deficiencies. In terms of algorithm efficiency, we should further reduce the number of redundant extensions and design more efficient heuristic techniques. Meanwhile, we can try to combine forward unfolding with reverse unfolding to integrate their advantages. In terms of data race detection, other static detection algorithms can be used to initially confirm the positions of statements where data race may occur, and then reverse unfolding algorithm is adopted for verification. Moreover, Reference [32] discussed robustness, compatibility, and deadlock detection of generalized concurrent systems. Subsequently, we will conduct studies on applications of reverse unfolding in these fields.

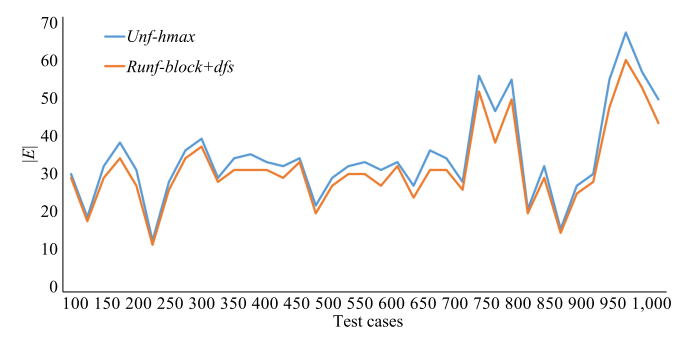

(a) Randomtree

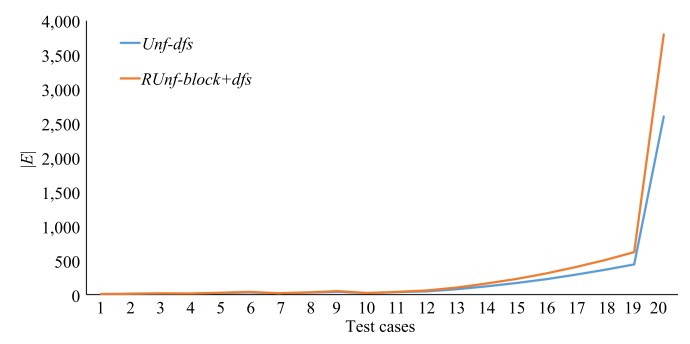

(b) Threadlock

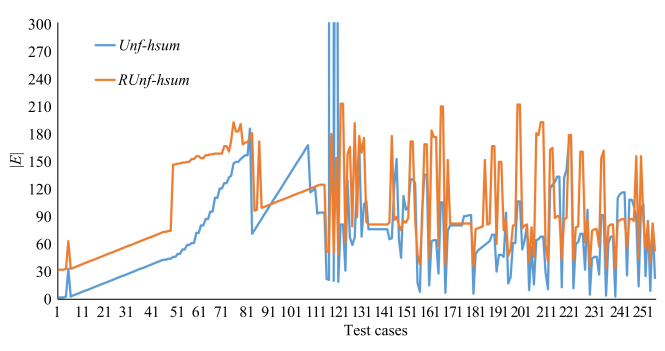

(c) Dartes

Figure 10 Comparison of $|E|$ in different test cases between directed unfolding and reverse unfolding

\section{References}

[1] Han L, Xing K, Chen X, Xiong F. A Petri net-based particle swarm optimization approach for scheduling deadlock-prone flexible manufacturing systems. Journal of Intelligent Manufacturing, 2018, 29(5): 1083-1096. [doi: 10.1007/s10845-015-1161-2].

[2] Hu L, Liu Z, Hu W, Wang Y, Tan J, Wu F. Petri-Net-Based dynamic scheduling of flexible manufacturing system via deep reinforcement learning with graph convolutional network. Journal of Manufacturing Systems, 2020, 55: 1-4. [doi: 10.1016/j.jmsy.2020.02.004].

[3] Fauzan AC, Sarno R, Yaqin MA. Performance measurement based on coloured Petri net simulation 
of scalable business processes. Proc. of the 2017 4th Int'l Conf. on Electrical Engineering, Computer Science and Informatics (EECSI). IEEE, 2017. 1-6. [doi: 10.1109/EECSI.2017.8239121].

[4] Liu C, Zeng Q, Duan H, Wang L, Tan J, Ren C, Yu W. Petri net based data-flow error detection and correction strategy for business processes. IEEE Access, 2020, 8: 43265-43276. [doi: 10.1109/ ACCESS.2020.2976124].

[5] Lu F, Tao R, Du Y, Zeng Q, Bao Y. Deadlock detection-oriented unfolding of unbounded Petri nets. Information Sciences, 2019, 497: 1-22. [doi: 10.1016/j.ins.2019.05.021].

[6] Xiang D, Liu G, Yan C, Jiang C. Detecting data inconsistency based on the unfolding technique of petri nets. IEEE Trans. on Industrial Informatics, 2017, 13(6): 2995-3005. [doi: 10.1109/TII.2017. 2698640].

[7] McMillan KL. Using unfoldings to avoid the state explosion problem in the verification of asynchronous circuits. Proc. of the Int'l Conf. on Computer Aided Verification. Berlin, Heidelberg: Springer-Verlag, 1992. 164-177.

[8] Engelfriet J. Branching processes of Petri nets. Acta Informatica, 1991, 28(6): 575-591. [doi: 10. 1007/BF01463946].

[9] Esparza J, Römer S, Vogler W. An improvement of McMillan's unfolding algorithm. Formal Methods in System Design, 2002, 20(3): 285-310. [doi: 10.1023/A:1014746130920].

[10] Khomenko V, Koutny M, Vogler W. Canonical prefixes of Petri net unfoldings. Acta Informatica, 2003, 40(2): 95-118. [doi: 10.1007/s00236-003-0122-y].

[11] Heljanko K, Khomenko V, Koutny M. Parallelisation of the Petri net unfolding algorithm. Proc. of the Int'l Conf. on Tools and Algorithms for the Construction and Analysis of Systems. Berlin, Heidelberg: Springer-Verlag, 2002. 371-385. [doi: 10.1007/3-540-46002-0_26].

[12] Benito FC, Kunzle LA. Unfolding for time Petri net. IEEE Latin America Transactions, 2017, 15(5): 1001-1008. [doi: 10.1109/TLA.2017.7912599].

[13] Schwarick M, Rohr C, Liu F, Assaf G, Chodak J, Heiner M. Efficient unfolding of coloured Petri nets using interval decision diagrams. Proc. of the Int'l Conf. on Applications and Theory of Petri Nets and Concurrency. Cham: Springer-Verlag, 2020. 324-344. [doi: 10.1007/978-3-030-51831-8_16].

[14] Dong L, Liu G, Xiang D. Verifying CTL with unfoldings of Petri nets. Proc. of the Int'l Conf. on Algorithms and Architectures for Parallel Processing. Cham: Springer-Verlag, 2018. 47-61. [doi: 1007/978-3-030-05063-4_5].

[15] Liu G, Reisig W, Jiang C, Zhou M. A branching-process-based method to check soundness of workflow systems. IEEE Access, 2016, 4: 4104-4118. [doi: 10.1109/ACCESS.2016.2597061].

[16] Chatain T, Paulevé L. Goal-Driven unfolding of Petri nets. arXiv: 1611.01296, 2016.

[17] Bonet B, Haslum P, Hickmott S, Thiébaux S. Directed unfolding of Petri nets. Proc. of the Trans. on Petri Nets and Other Models of Concurrency I. Berlin, Heidelberg: Springer-Verlag, 2008. 172-198. [doi: 10.1007/978-3-540-89287-8_11].

[18] Bonet B, Haslum P, Khomenko V, Thiébaux S, Vogler W. Recent advances in unfolding technique. Theoretical Computer Science, 2014, 551: 84-101. [doi: 10.1016/j.tcs.2014.07.003].

[19] Abdulla PA, Iyer SP, Nylén A. SAT-Solving the coverability problem for Petri nets. Formal Methods in System Design, 2004, 24(1): 25-43. [doi: 10.1023/B:FORM.0000004786.30007.f8].

[20] Leveson NG, Turner CS. An investigation of the Therac-25 accidents. Computer, 1993, 26(7): 18-41. [doi: 10.1109/MC.1993.274940].

[21] Poulsen K. Software bug contributed to blackout. Security Focus. 2004. http://www.securityfocus. com/news/8016.

[22] Joab J. Nasdaq's Facebook glitch came from 'race conditions'. 2012. http://www.computerworld.com/ s/article/9227350.

[23] Blackshear S, Gorogiannis N, O’Hearn PW, Sergey I. RacerD: Compositional static race detection. Proc. of the ACM on Programming Languages, 2018.2(OOPSLA): 1-28. [doi: 10.1145/3276514].

[24] Chatarasi P, Shirako J, Kong M, Sarkar V. An extended polyhedral model for SPMD programs and its use in static data race detection. Proc. of the Int'1 Workshop on Languages and Compilers for Parallel 
Computing. Cham: Springer-Verlag, 2016. 106-120. [doi: 1007/978-3-319-52709-3_10]

[25] Bora U, Das S, Kukreja P, Joshi S, Upadrasta R, Rajopadhye S. Llov: A fast static data-race checker for OpenMP programs. ACM Trans. on Architecture and Code Optimization (TACO), 2020, 17(4): 1-26. [doi: 10.1145/3418597].

[26] Wilcox JR, Flanagan C, Freund SN. VerifiedFT: A verified, high-performance precise dynamic race detector. Proc. of the 23rd ACM SIGPLAN Symp. on Principles and Practice of Parallel Programming. 2018. 354-367. [doi: 1145/3178487.3178514].

[27] Gu Y, Mellor-Crummey J. Dynamic data race detection for OpenMP programs. Proc. of the SC18: Int'1 Conf. for High Performance Computing, Networking, Storage and Analysis. IEEE, 2018. 767-778. [doi: 10.1109/SC.2018.00064].

[28] Lidbury C, Donaldson AF. Dynamic race detection for C++ 11. ACM SIGPLAN Notices, 2017, 52(1): 443-457. [doi: 1145/3093333.3009857].

[29] Kavi KM, Moshtaghi A, Chen DJ. Modeling multithreaded applications using Petri nets. Int'1 Journal of Parallel Programming, 2002, 30(5): 353-371. [doi: 10.1023/A:1019917329895].

[30] Vallée-Rai R, Co P, Gagnon E, Hendren L, Lam P, Sundaresan V. Soot: A Java bytecode optimization framework. Proc. of the CASCON 1st Decade High Impact Papers. 2010. 214-224. [doi: 10.1145/ 1925805.1925818].

[31] https://github.com/Zongyin-Hao/Coverability

[32] Liu GJ. Meta-unfolding of Petri Nets: A Model Checking Method of Concurrent Systems. Beijing: Science Press, 2020 (in Chinese).

\section{Appendix A}

First, we provide the definition of adequate order. A partial order $\prec$ is an adequate order if it satisfies

(1) $\prec$ is well-founded;

(2) $\prec$ is refinement of $\subset, C f g_{1} \subset C f g_{2}$ means $C f g_{1} \prec C f g_{2}$;

(3) If $\operatorname{Mark}\left(C f g_{1}\right) \leq \operatorname{Mark}\left(C f g_{2}\right), C f g_{1} \prec C f g_{2}$, then for any prefix $E_{2}$ of $C f g_{2}$, there is $E_{1}$ satisfying $\operatorname{Mark}\left(C f g_{1} \oplus E_{1}\right) \leq \operatorname{Mark}\left(C f g_{2} \oplus E_{2}\right)$ and $C f g_{1} \oplus E_{1} \prec C f g_{2} \oplus E_{2}$.

Theorem 1. $\prec_{r}$ is an adequate order.

Proof: It is obvious that $\prec_{r}$ satisfies conditions (1) and (2) of adequate order, and we only need to prove $\prec_{r}$ satisfies condition (3). Specifically, we need to prove when $\operatorname{Mark}\left(\operatorname{Cfg}_{1}\right) \leq$ $\operatorname{Mark}\left(C f g_{2}\right) \wedge C f g_{1} \prec_{r} C f g_{2}$, for any prefix $E_{2}$ of $C f g_{2}$, there is $E_{1}$ satisfying $\operatorname{Mark}\left(C f g_{1} \oplus\right.$ $\left.E_{1}\right) \leq \operatorname{Mark}\left(C f g_{2} \oplus E_{2}\right)$ and $C f g_{1} \oplus E_{1} \prec_{r} C f g_{2} \oplus E_{2}$.

$E_{k}$ is defined as a prefix of the configuration $C f g$ with a size of $k, C f g^{k}=C f g \oplus E^{k}$. Then we develop proof by induction according to $\left|E_{2}\right|$. When $\left|E_{2}\right|=0$, the conclusion is established obviously. When $\left|E_{2}\right|=k, \operatorname{Mark}\left(C f g_{1}^{k}\right) \leq \operatorname{Mark}\left(C f g_{2}^{k}\right)$ and $C f g_{1}^{k} \prec_{r} C f g_{2}^{k}$ can be obtained from the inductive assumption. When $\left|E_{2}\right|=k+1$, for any transition $t$ let $e_{2}=\left(t, C_{2}\right), \mu\left(C_{2}\right) \subseteq t \wedge C_{2} \subseteq C u t\left(C f g_{2}^{k}\right)$, so $C f g_{2}^{k+1}=C f g_{2}^{k} \cup\left\{e_{2}\right\}$; let $e_{1}=\left(t, C_{1}\right)$, $\mu\left(C_{1}\right) \subseteq t \wedge C_{1} \subseteq \operatorname{Cut}\left(C f g_{1}^{k}\right)$, so $C f g_{1}^{k+1}=C f g_{1}^{k} \cup\left\{e_{1}\right\} . \mu\left(C_{1}\right) \leq \mu\left(C_{2}\right)$ is required and $C_{1}$ should be as big as possible $\left(C_{1}\right.$ can be $\left.\varnothing\right)$.

Then we only need to prove $\operatorname{Mark}\left(C f g_{1}^{k+1}\right) \leq \operatorname{Mark}\left(C f g_{2}^{k+1}\right)$ and $C f g_{1}^{k+1} \prec_{r} C f g_{2}^{k+1}$. There are the following two situations.

(1) $C_{1}=\varnothing: C f g_{1}^{k}=C f g_{1}^{k+1}$. As it is required that $C_{1}$ should be as big as possible, it can be known that $\forall p \in \mu\left(C_{2}\right), p \notin \operatorname{Mark}\left(C f g_{1}^{k}\right)$. Thus, it can be obtained that $\operatorname{Mark}\left(C f g_{1}^{k+1}\right)=$ $\operatorname{Mark}\left(C f g_{1}^{k}\right) \leq \operatorname{Mark}\left(C f g_{2}^{k}\right)-\mu\left(C_{2}\right)+t=\operatorname{Mark}\left(C f g_{2}^{k+1}\right)$. In addition, due to $\left|C f g_{2}^{k}\right|<$ $\left|C f g_{2}^{k+1}\right|$, according to the definition (1) of $\prec_{r}, C f g_{2}^{k} \prec C f g_{2}^{k+1}$, and thereby $C f g_{1}^{k+1}=$ $C f g_{1}^{k} \prec_{r} C f g_{2}^{k} \prec_{r} C f g_{2}^{k+1}$. Thus, the conclusion holds when $C_{1}=\varnothing$. 
(2) $C_{1} \neq \varnothing$ : As it is required that $C_{1}$ should be as big as possible and $\mu\left(C_{1}\right) \leq \mu\left(C_{2}\right)$, it can be known that $\forall p \in \mu\left(C_{2}\right)$, and $p \in \mu\left(C_{1}\right) \vee p \notin \operatorname{Mark}\left(C f g_{1}^{k}\right)$. Thus, one can obtain $\operatorname{Mark}\left(C f g_{1}^{k+1}\right)=\operatorname{Mark}\left(C f g_{1}^{k}\right)-\mu\left(C_{1}\right)+t \leq \operatorname{Mark}\left(C f g_{2}^{k}\right)-\mu\left(C_{2}\right)+t=\operatorname{Mark}\left(C f g_{2}^{k+1}\right)$. When $\left|C f g_{1}^{k}\right|<\left|C f g_{2}^{k}\right|$, there is $\left|C f g_{1}^{k+1}\right|=\left|C f g_{1}^{k}\right|+1<\left|C f g_{2}^{k}\right|+1=\left|C f g_{2}^{k+1}\right|, C f g_{1}^{k+1} \prec_{r} C f g_{2}^{k+1}$. When $\left|C f g_{1}^{k}\right|=\left|C f g_{2}^{k}\right|$ and $\operatorname{Lex}\left(\mu\left(C f g_{1}^{k}\right)\right)<\operatorname{Lex}\left(\mu\left(C f g_{2}^{k}\right)\right)$, there is $\left|C f g_{1}^{k+1}\right|=\left|C f g_{1}^{k}\right|+1<$ $\left|C f g_{2}^{k}\right|+1=\left|C f g_{2}^{k+1}\right|$. After two ordered strings with the same size are added with the same element and are re-ordered, their precedence relationship in the lexicographical order will not change, so $\operatorname{Lex}\left(\mu\left(C f g_{1}^{k+1}\right)\right)<\operatorname{Lex}\left(\mu\left(C f g_{2}^{k+1}\right)\right), C f g_{1}^{k+1} \prec_{r} C f g_{2}^{k+1}$. Thus, the conclusion is established when $C_{1} \neq \varnothing$.

To sum up, $\operatorname{Mark}\left(C f g_{1} \oplus E_{1}\right) \leq \operatorname{Mark}\left(C f g_{2} \oplus E_{2}\right)$ and $C f g_{1} \oplus E_{1} \prec_{r} C f g_{2} \oplus E_{2}$. So $\prec_{r}$ is an adequate order.

Theorem 2. Reverse cut-off events will not break the completeness of $R U n f$.

Proof: For any reachable marking $M$ satisfying $M \mapsto M_{f}$, there should be a configuration $C f g$ satisfying $\operatorname{Mark}(C f g) \subseteq M$. Assuming $C f g$ is not included in $R U n f, C f g$ must contain a reverse cut-off event $e$, and there is an event $e^{\prime}$ in $R U n f$ satisfying $\operatorname{Mark}\left(\left[e^{\prime}\right]\right) \leq \operatorname{Mark}([e]) \wedge\left[e^{\prime}\right] \prec$ $[e]$.

According to the condition (3) of adequate order, for $C f g=[e] \oplus E$, there is $C f g^{\prime}=\left[e^{\prime}\right] \oplus E^{\prime}$ satisfying $\operatorname{Mark}\left(C f g^{\prime}\right) \leq \operatorname{Mark}(C f g) \leq M \wedge C f g^{\prime} \prec C f g$. As $\prec$ is well-founded, the above process will finally find a minimum configuration in $R U n f$, and this is not consistent with the assumption. Thus, reverse cut-off events will not break the completeness of $R U n f$.

Theorem 3. RUnf is finite.

Proof: For any event $e$ in RUnf, there is a longest chain $e_{1}<e_{2}<\cdots<e$, whose length is set as $d(e)$. The following three conclusions are drawn.

(1) For any condition $c,{ }^{\bullet} c$ and $c^{\bullet}$ are finite. For any event $e,{ }^{\bullet} e$ and $e^{\bullet}$ are finite.

(2) Let the number of reachable markings of a 1-safe Petri net be $n$; and there is $d(e) \leq n+1$. For a chain $e_{1}<e_{2}<\cdots<e_{n+1}$ with a size of $n+1$, there are definitely two events $e_{i}$ and $e_{j}(i<j)$ which satisfy $\operatorname{Mark}\left(\left[e_{i}\right]\right) \leq \operatorname{Mark}\left(\left[e_{j}\right]\right)$. In addition, due to $\left[e_{i}\right] \subset\left[e_{j}\right],\left[e_{i}\right] \prec\left[e_{j}\right]$ are obtained according to the condition (2) of adequate order, where $e_{j}$ is a reverse cut-off event. Thus there is no $e$ in the chain which satisfies $e_{j}<e$, namely that the length of the chain cannot be greater than $n+1$.

(3) For any $k \geq 0$, RUnf only includes finite event $e$ satisfying $d(e) \leq k$.

It can be proved inductively that when $k=0$, the conclusion holds obviously. Let $E_{k}$ be the event set when $d(e) \leq k$, and the inductive assumption yields that $E_{k}$ is finite. $E_{k+1}$ is the event set when $d(e) \leq k+1$. In light of $E_{k+1}^{\bullet} \subseteq{ }^{\bullet} E_{k} \cup C M_{f}$ and conclusion (1), one can know $E_{k+1}$ is finite.

Conclusions (2) and (3) indicate that $R U n f$ includes finite events, and conclusion (1) shows that $R U n f$ includes finite conditions, so $R U n f$ is finite.

\section{Appendix B}

First, let us review the two conditions of RExt.

(1) For any event $e$ in $R U n f$, there is no reverse extension rext $=(t, C)$ in $R E x t$ which satisfies $\mu(e)=t \wedge e^{\bullet}=C$.

(2) For any two different reverse extensions rext $t_{1}=\left(t_{1}, C_{1}\right)$ and rext $t_{2}=\left(t_{2}, C_{2}\right)$ in RExt, if $t_{1}=t_{2} \wedge C_{1} \subset C_{2} \wedge \operatorname{Mark}\left(\left[\operatorname{rext}_{1}\right]\right) \geq \operatorname{Mark}\left(\left[\operatorname{rext}_{2}\right]\right)$, the extension $\operatorname{rext}_{1}$ is deleted from RExt. 
Parosh first proposed the principle of reverse unfolding in Reference [19], but did not add the constraint $\operatorname{Mark}\left(\left[\operatorname{rext}_{1}\right]\right) \geq \operatorname{Mark}\left(\left[\operatorname{rext}_{2}\right]\right)$ to the extension rule, which thus results in the incompleteness of reverse unfolding. Here we analyze the counterexample.

We use the Petri net shown in Figure 11 and its reverse unfolding to illustrate. The left shows the original Petri net, and the target marking is $\{p 12\}$. Obviously, the target marking is coverable. The right shows the reverse unfolding without the constraint $\operatorname{Mark}\left(\left[\operatorname{rext}_{1}\right]\right) \geq \operatorname{Mark}\left(\left[\operatorname{rext}_{2}\right]\right)$. When the reverse unfolding algorithm generates the event $e 8$, the corresponding reverse extension is rext $1=(t 8,\{c 2, c 9\})$, and rext $2=(t 8,\{c 2\})$ will be deleted according to Parosh's rule. However, when analyzing the original Petri net, we find that the reverse marking $\{p 4, p 8\}$ corresponding to rext 1 is uncoverable, and the reverse marking $\{p 8, p 11\}$ corresponding to rext 2 is coverable. Further, the reverse marking corresponding to rext 2 is necessary for determining the coverability of $\{p 12\}$. At this point, it is impossible to verify the coverability of the target marking regardless of subsequent extensions, and the completeness of $R U n f$ has been broken.
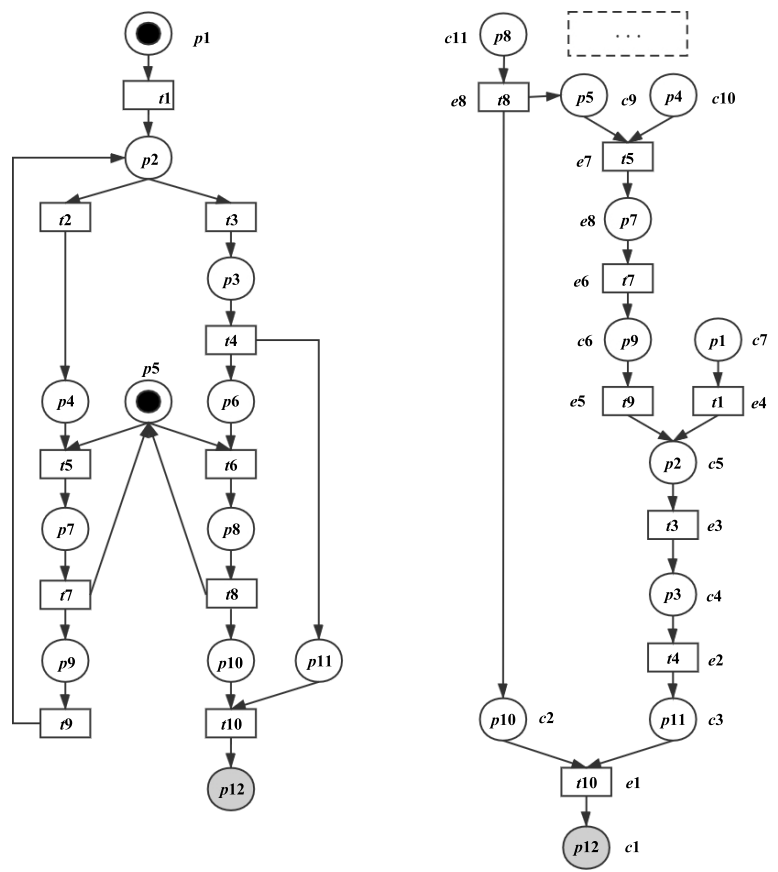

Figure 11 Example on completeness destruction of reverse unfolding

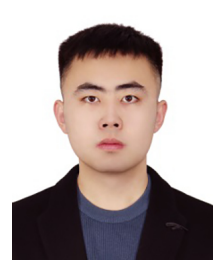

Zongyin Hao, master degree candidate, $\mathrm{CCF}$ student member. His research interests include the theory and applications of Petri nets and formal verification of concurrent program.

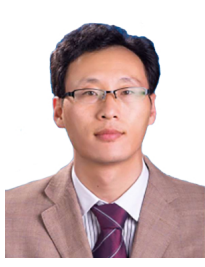

Faming Lu, Ph.D., associate professor, doctoral supervisor, CCF professional member. His research interests include the theory and applications of Petri nets, modeling and analysis of concurrent systems, business process management, and decision support. 